\title{
Numerical simulation of the wake of marine current turbines with a particle method
}

\author{
Grégory Pinon ${ }^{\mathrm{a}, *}$, Paul Mycek ${ }^{\mathrm{a}, \mathrm{b}}$, Grégory Germain ${ }^{\mathrm{b}}$, Elie Rivoalen ${ }^{\mathrm{c}, \mathrm{a}}$ \\ a Laboratoire Ondes et Milieux Complexes, UMR 6294, CNRS-Université du Havre 53, rue de Prony, BP540, F- \\ 76058 Le Havre Cedex, France \\ b IFREMER, Hydrodynamic \& Metocean Service 150, Quai Gambetta, BP699, F-62321 Boulogne-Sur-Mer, \\ France \\ ${ }^{c}$ Laboratoire d'Optimisation et Fiabilité en Mécanique des Structures, EA 3828, INSA de Rouen Avenue de \\ l'Université, BP 08, \\ *: Corresponding author : Grégory Pinon, Tel.: +33 235217123 ; email address : gregory.pinon@univ-lehavre.fr
}

\begin{abstract}
:
This paper presents numerical computations of three bladed horizontal axis marine current turbines in a uniform free upstream current. The unsteady evolution of the turbine wake is taken into account by some three-dimensional software, developed to assess the disturbances generated in the sea. An unsteady Lagrangian method is considered for these computations using "Vortex Method"; a velocityvorticity numerical implementation of the Navier-Stokes equations. The vortex flow is discretised with particles carrying vorticity, which are advected in a Lagrangian frame. The present paper aims at presenting results on both power and thrust coefficient $\left(C_{P}\right.$ and $\left.C_{T}\right)$ predictions and wake characterisation, up to ten diameters downstream of the turbine. Moreover, two different marine current turbines configurations are considered: one is taken from literature [1] and the second one is an open-modified version of turbine inspired from previous works [2].
\end{abstract}

\section{Highlights}

- We developed a numerical software for the computation of marine current turbine. We used an unsteady Lagrangian implementation of the Navier-Stokes equations. Two different turbines are considered, one of them is taken from the literature [1]. Both power/thrust coefficients and wake are computed with the same numerical tool. All the numerical results are compared to experiments

Keywords: Numerical computations ; Marine current turbine ; Hydrodynamic ; Power and thrust coefficients ; Wake

\section{Introduction}

At the present time, many studies are being carried out concerning the energy resources available from tides or currents in the oceans around Europe [3,4] and even around China [5, 6], one of the first being [7] for the U.K. However, with the future large deployment of marine current energy converters, many questions have been raised about their impact on the environment $[8,9,10]$. Modifications of the overall flow patterns in the area of current energy devices [9] may alter the erosion and sediment transport by their wake effects [11], and even the free surface of the sea [12, 13]. There is concern that even a small change to these processes could cause significant environmental impacts. For these reasons, there is a need for numerical prediction of the overall flow modification prior to the installation of a marine current farm.

Concerning horizontal axis marine current turbines, Computational Fluid Dynamics (CFD) has already been used for power and thrust coefficients $\left(C_{P}\right.$ and $\left.C_{T}\right)$ 
predictions $[1,12,14,15]$. In most of these studies, the Blade Element Momentum theory (BEM) was used and all the numerical results were compared to experimental values issuing from [16]. In the present paper, $C_{P}$ and $C_{T}$ are also compared to the same experimental results [16] as a matter of validation. However, in the previous studies, the wake was not evaluated except for [15] where the wake was used for a better numerical evaluation of power and thrust coefficients.

CFD has also been used for wake computations under the actuator disc approximation $[17,13,18,19,20]$. In this manner, comparisons with experiments are reasonable only if the numerical wakes are compared to the wakes behind a disc $[17,18]$. It is actually very different from the present approach where the unsteady flow behind the turbine is computed. In addition, under the actuator disc approximation, no real $C_{P}$ and $C_{T}$ evaluation is possible. To the authors' knowledge, the few numerical studies that treat both $C_{P} / C_{T}$ and wake characterisation mostly refer to vertical axis marine current turbines [21, 22]. Unfortunately, in the two latter studies, there is no real comparison with experimental data.

The aim of the present paper is to present computations of a three bladed horizontal axis marine current turbine. Both power and thrust coefficients $\left(C_{P}\right.$ and $\left.C_{T}\right)$ predictions and wake characterisation are presented and discussed. To this purpose, three-dimensional software, initially issuing from $[23,24]$ is being developed in order to assess the disturbances generated in the sea. This is an unsteady Lagrangian frame software designed to compute the evolution of the wake emitted by the turbine. This study can be viewed as a extension of a previous study [25]. In the first part, the governing equations and the numerical implementations are presented. Then, in the second section, the two different marine current turbine configurations are detailed. The first is taken from literature $[1,16,14]$ and the second configuration is an open-modified version of turbine inspired from previous studies carried out by the same team of researchers [2]. The last two sections present the numerical results and, as far as possible, these results are compared to related experimental data in order to validate the numerical tools. Furthermore, some convergence analyses of the method are presented and discussed. The third part is dedicated to power and thrust coefficients $\left(C_{P}\right.$ and $\left.C_{T}\right)$ evaluation for the two different blade configurations. Finally, the last section deals with wake characterisation: $2 \mathrm{D}$ velocity maps, velocity profiles and axial velocity decay in the wake of a three-bladed turbine.

\section{Numerical methods}

An unsteady Lagrangian method is used for these simulations using a Vortex Method. It is considered that the flow is discretised with vorticity-carrying particles, which are advected in a Lagrangian frame. The particles are emitted at the trailing edge of the turbine blades thanks to a panel method with dipoles using the KuttaJoukovski condition as an emission scheme. 


\subsection{Governing equations}

The flow of an incompressible fluid is governed by the Navier-Stokes equations, which are written in velocity-vorticity formulation $(\mathbf{u}, \boldsymbol{\omega})$ :

$$
\begin{aligned}
\nabla \cdot \mathbf{u} & =0 \\
\frac{D \boldsymbol{\omega}}{D t} & =(\boldsymbol{\omega} \cdot \boldsymbol{\nabla}) \mathbf{u}+\nu \Delta \boldsymbol{\omega}
\end{aligned}
$$

where $\boldsymbol{\omega}=\boldsymbol{\nabla} \wedge \mathbf{u}$ is the vorticity field of the flow, $\mathbf{u}$ is the velocity field and $D / D t$ is the material derivative. Equation (1) stands for the mass conservation equation in an incompressible fluid and equation (2) is the transport equation of vorticity. $D \boldsymbol{\omega} / D t$ represents the transport term in a Lagrangian frame, $(\boldsymbol{\omega} \cdot \boldsymbol{\nabla}) \mathbf{u}$ stands for the stretching term, which disappears in two dimensions and $\nu \Delta \boldsymbol{\omega}$ represents the diffusion. The Helmholtz decomposition of the velocity field gives:

$$
\mathbf{u}=\boldsymbol{\nabla} \wedge \boldsymbol{\psi}+\boldsymbol{\nabla} \phi=\mathbf{u}^{\psi}+\mathbf{u}^{\phi}
$$

where $\boldsymbol{\psi}$ is a vector potential and $\phi$ is a scalar potential. With equations (1) and (3), we obtain:

$$
\begin{aligned}
\Delta \boldsymbol{\psi} & =-\boldsymbol{\omega} \\
\Delta \phi & =0
\end{aligned}
$$

The velocity field $\mathbf{u}$ can be expressed as the sum of three components, $\mathbf{u}^{\psi}$ the rotational component of the velocity field, $\mathbf{u}^{\phi}$ the potential velocity field, and $\mathbf{u}^{\infty}$ a constant uniform velocity representing, in the present configuration, the marine current inflow. We finally have:

$$
\mathbf{u}=\mathbf{u}^{\psi}+\mathbf{u}^{\phi}+\mathbf{u}^{\infty}
$$

The rotational component of the velocity field

From equation (4), for any point $M$ of the fluid domain $\mathcal{D}$, the rotational component $\mathbf{u}^{\psi}=\boldsymbol{\nabla} \wedge \boldsymbol{\psi}$ of the velocity field can be evaluated thanks to the Biot-Savart law:

$$
\mathbf{u}^{\psi}(M)=\frac{1}{4 \pi} \int_{v} \mathbf{K}\left(\mathbf{M M}^{\prime}\right) \wedge \boldsymbol{\omega}\left(\mathbf{M}^{\prime}\right) d v^{\prime},
$$

with $\mathbf{K}\left(\mathbf{M M}^{\prime}\right)=\frac{\mathbf{M M}^{\prime}}{\left|\mathbf{M M}^{\prime}\right|^{3}}$. In order to prevent the computations collapsing owing to the singular behaviour of $\mathbf{K}$ when two particles are getting too close, a desingularised kernel $\mathbf{K}_{\varepsilon}$ is used (eq. (8)) as in Lindsay \& Krasny [26].

$$
\mathbf{K}_{\varepsilon}\left(\mathbf{M M}^{\prime}\right)=\frac{\mathbf{M M}^{\prime}}{\left(\left|\mathbf{M M}^{\prime}\right|^{2}+\varepsilon^{2}\right)^{3 / 2}}
$$

This desingularised kernel is commonly used in the Lagrangian vortex community; several references can be consulted on this particular topic, amongst others in Cottet \& Koumoutsakos [27]. The so-called smoothing parameter $\varepsilon$ is equivalent to the characteristic mesh size in Eulerian frame computations. A commonly wide spread value of $\varepsilon$ is 1.5 times the characteristic inter-particle spacing. 
The first step consists in discretising the vorticity field into vortex elements, which are indifferently termed either blobs or particles hereafter. A particle $P_{i}$ physically represents the vortical barycentre of a small volume of fluid $d v$. The position $\mathbf{X}_{i}$ of particle $P_{i}$ and the vorticity $\Omega_{i}$ carried by this particle are respectively defined by:

$$
\mathbf{X}_{i}=\frac{\int_{P_{i}} \mathbf{x} d v}{\int_{P_{i}} d v} \text { and } \boldsymbol{\Omega}_{i}=\int_{P_{i}} \boldsymbol{\omega} d v .
$$

The particles' emission process is described in details in following section 2.3 and the time integration scheme in section 2.2. At a given location $\mathbf{X}_{i}$ of the fluid domain $\mathcal{D}$, the discrete rotational component $\mathbf{U}_{i}^{\psi}=\mathbf{u}^{\psi}\left(\mathbf{X}_{i}\right)$ is derived from equation (7) as follows:

$$
\mathbf{U}_{i}^{\psi}=\mathbf{u}^{\psi}\left(\mathbf{X}_{i}\right)=\frac{1}{4 \pi} \sum_{j=1}^{N} \mathbf{K}_{\varepsilon}\left(\mathbf{X}_{i}-\mathbf{X}_{j}\right) \wedge \boldsymbol{\Omega}_{j}
$$

where $N$ represents the total number of particles. Of course, the computation being unsteady, as the wake develops, the total number of particles increases with time $(N=N(t))$. However, the time dependence of $N$ will not be written as a matter of simplification. In addition, the Tree Code algorithm developed by Lindsay \& Krasny [26] has been implemented. This Tree Code is based on a Taylor expansion on the desingularised kernel $\mathbf{K}_{\varepsilon}$ in order to speed up the determination of the velocity $\mathbf{U}_{i}^{\psi}$ (eq. (10)). The Tree Code performances in the present implementation can be found in $[24]$.

\section{The potential velocity}

From equation (5), for any point $M$ of the fluid domain $\mathcal{D}$, the potential velocity $\mathbf{u}^{\phi}=\boldsymbol{\nabla} \phi$ is defined in order to take into account the turbine blades $\mathcal{S}$ :

$$
\left\{\begin{aligned}
\frac{\partial \phi}{\partial \mathbf{n}}(P) & =-\mathbf{v}(P) \cdot \mathbf{n}(P) \quad \forall P \in \mathcal{S} \\
\Delta \phi(M) & =0 \quad \forall M \in \mathcal{D} \\
\lim _{|\mathbf{M P}| \rightarrow+\infty} \boldsymbol{\nabla} \phi & =0 \quad \forall M \in \mathcal{D}, \forall P \in \mathcal{S}
\end{aligned}\right.
$$

with $\mathbf{v}=\mathbf{u}-\mathbf{u}^{\phi}=\mathbf{u}^{\psi}+\mathbf{u}^{\infty}$ the residual velocity in the case of a motionless turbine. For rotating blades, an additional velocity component must to be defined as it will be presented hereafter (cf. eq. (19)). The slip velocity condition (eq. (11a)) is imposed at every control point $P \in \mathcal{S}$ of the turbine. Moreover, the potential velocity $\mathbf{u}^{\phi}$ induced by these boundaries must satisfy the two last conditions, equation (11b) initially issuing from the solenoidal condition (1) and the perturbation velocity decay far from the turbine (eq. (11c)). This velocity potential $\phi$ is calculated using equations (11) and Green's third identity:

$$
\phi(M)=\frac{1}{4 \pi} \iint_{\mathcal{S}} \mu(P) \frac{\mathbf{M P} \cdot \mathbf{n}(P)}{|\mathbf{M P}|^{3}} d s,
$$

$\mu(P)$ representing a distribution of normal dipoles on the turbine blade surface $\mathcal{S}$. Assuming that the turbine blades are discretised into $N_{P}$ surface elements with normal vectors $\mathbf{n}_{p}$ and elementary surfaces $d s_{p}$, that the dipoles distribution $\mu(P)$ is 
constant onto a surface element and equal to $\mu_{p}$, the semi-discrete velocity component $\mathbf{u}^{\phi}(M)$ is defined as:

$$
\mathbf{u}^{\phi}(M)=\frac{1}{4 \pi} \sum_{p=1}^{N_{p}} \mu_{p} \nabla_{M}\left(\frac{\mathbf{M P} \cdot \mathbf{n}(P)}{|\mathbf{M P}|^{3}}\right) d s_{p}=-\frac{1}{4 \pi} \sum_{p=1}^{N_{p}} \mu_{p} \int_{\mathcal{C}_{p}} \nabla_{M}\left(\frac{1}{\mathrm{MP}}\right) \wedge \mathrm{dl},
$$

whose last formulation, on the right hand side of previous equation (13), comes from [28]. We obtain the final discrete formulation of $\mathbf{U}_{i}^{\phi}=\mathbf{u}^{\phi}\left(\mathbf{X}_{i}\right)$ by basically replacing the $\mathcal{C}_{p}$ curvilinear integral by the contribution of the four segments $\mathbf{l}_{p}^{n}(n=1, \ldots, 4)$ of the $p^{t h}$ facet:

$$
\mathbf{U}_{i}^{\phi}=\mathbf{u}^{\phi}\left(\mathbf{X}_{i}\right)=\frac{1}{4 \pi} \mu_{p} \sum_{n=1}^{4} \boldsymbol{\alpha}_{i p}^{n}
$$

with

$$
\boldsymbol{\alpha}_{i p}^{n}=\frac{\mathbf{r}_{i p}^{n_{1}} \wedge \mathbf{r}_{i p}^{n_{2}}}{\left|\mathbf{r}_{i p}^{n_{1}} \wedge \mathbf{r}_{i p}^{n_{2}}\right|^{2}}\left[\left|\mathbf{r}_{i p}^{n_{1}}\right|+\left|\mathbf{r}_{i p}^{n_{2}}\right|\right]\left[1-\frac{\mathbf{r}_{i p}^{n_{1}} \cdot \mathbf{r}_{i p}^{n_{2}}}{\left|\mathbf{r}_{i p}^{n_{1}}\right|\left|\mathbf{r}_{i p}^{n_{2}}\right|}\right]
$$

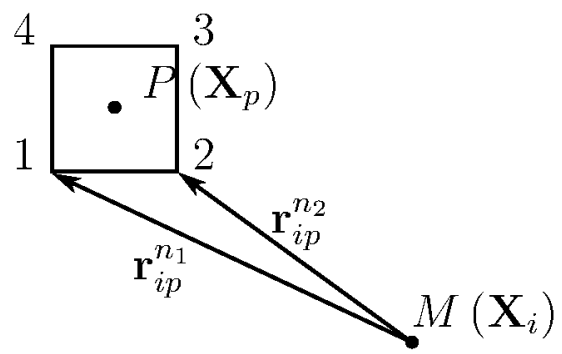

Figure 1: Representation of the $\mathbf{r}_{i p}^{n_{k}}(k \in\{1 ; 2\})$

Figure 1 can be refered to for geometrical details. In order to determine the normal dipoles distribution $\mu_{p}$ on the blade surface $\mathcal{S}$, equation (11a) can be rewritten for each control point $P\left(\mathbf{X}_{p}\right)$ of the turbine, assuming that $\mathbf{V}_{p}=\mathbf{v}\left(\mathbf{X}_{p}\right)$ :

$$
\mathbf{U}_{p}^{\phi} \cdot \mathbf{n}_{p}=-\mathbf{V}_{p} \cdot \mathbf{n}_{p}, \quad p=1, \cdots, N_{P} .
$$

Solving this previous equation for all control points $P\left(\mathbf{X}_{p}\right) \in \mathcal{S}$ is equivalent to defining a linear matrix system:

$$
[\mathbf{A}][\boldsymbol{\mu}]=[\mathbf{S M}]
$$

where

$[$ A $]$ is a matrix obtained using equation (18):

$$
a_{q p}=\frac{\mathbf{n}_{q}}{4 \pi} \cdot \sum_{n=1}^{4} \boldsymbol{\alpha}_{q p}^{n}
$$

with $\boldsymbol{\alpha}_{q p}^{n}$ evaluated with the help of equation (15). This term physically represents the influence of the $p^{\text {th }}$ element (resp. $\mu_{p}$ dipole) onto the $q^{\text {th }}$ element (resp. $\mu_{q}$ dipole). This so-called influence matrix [A] only depends on the turbine surface mesh geometry and can therefore be reversed and stored once at the beginning of the computation. 
$[\boldsymbol{\mu}]$ is an unknown vector representing the dipoles distribution $\mu_{p}\left(p=1, \cdots, N_{P}\right)$.

$[\mathbf{S M}]$ is the second member of the matrix system and physically represents the residual velocity we want to annihilate on the blade control points $P\left(\mathbf{X}_{p}\right)$ in order to enforce the slip condition (eq. (16)). However, as explained in section 2.3, the emitted particles mainly depend upon the bounded vortex sheet strength $\gamma$ (eq. (36)), which is closely linked to the normal dipoles distribution (eq. (38)). In the case of a moving obstacle, the bounded vortex sheet strength has to be evaluated in the moving frame (i.e. the rotating frame). Therefore, these residual velocities consist of the rotational velocity $\mathbf{u}^{\psi}$ due to the presence of the particles, the main stream velocity $\mathbf{u}^{\infty}$ and the blades' rotation velocity $\mathbf{u}^{\text {rot }}(\mathbf{r})=\boldsymbol{\Phi} \wedge \mathbf{r}$, with $\boldsymbol{\Phi}$ the turbine rotation vector and $\mathbf{r}$ the radius between the concerned location and the rotation centre. $[\mathbf{S M}]$ is then basically obtained from equation (11a), modified in order to be expressed in the rotating frame, and reads:

$$
[\mathbf{S M}]=\mathbf{U}_{p}^{\phi} \cdot \mathbf{n}_{p}=-\mathbf{V}_{\mathbf{p}} \cdot \mathbf{n}_{p}
$$

with $\mathbf{V}_{p}=\mathbf{U}_{p}^{\psi}+\mathbf{U}_{p}^{\infty}-\mathbf{U}_{p}^{\text {rot }}$ the residual velocity in the case of a rotating turbine.

\subsection{Numerical scheme}

Thanks to the Helmholtz decomposition (eq. (3) and (6)), the discrete velocity $\mathbf{U}_{i}=\mathbf{u}\left(\mathbf{X}_{i}\right)$ at a given location $\mathbf{X}_{i}$ of the flow in the Galilean frame $\mathcal{R}_{g}$ is basically obtained by the following sum:

$$
\mathbf{U}_{i}=\mathbf{U}_{i}^{\psi}+\mathbf{U}_{i}^{\phi}+\mathbf{U}_{i}^{\infty}
$$

where

$\mathbf{U}_{i}^{\infty}$ is the infinite upstream velocity field, which is reduced in the present paper to a constant velocity,

$\mathbf{U}_{i}^{\psi}$ is the velocity field induced by the vortical wake, obtained by equation (10),

$\mathbf{U}_{i}^{\phi}$ is the potential velocity field induced by the normal dipoles distribution $\mu$ in order to enforce a free-slip velocity condition onto the blades, obtained by equation (14).

The discrete evolution of the vortical particles $\left(\mathbf{X}_{i}, \boldsymbol{\Omega}_{i}\right)$ is given by the transport equation of the particles positions $\mathbf{X}_{i}$ by their own velocities $\mathbf{U}_{i}$ as presented in equation (21):

$$
\begin{aligned}
\frac{D \mathbf{X}_{i}}{D t} & =\mathbf{U}_{i} \\
\frac{D \boldsymbol{\Omega}_{i}}{D t} & =\left(\boldsymbol{\Omega}_{i} \cdot \boldsymbol{\nabla}\right) \mathbf{U}_{i}+\nu \Delta \boldsymbol{\omega}_{\left(\mathbf{x}=\mathbf{X}_{i}\right)} \mathcal{V}_{i}
\end{aligned}
$$

Equation (22) is the transport equation of the vortex particles $\Omega_{i}$ and represents a discrete version of the Navier-Stokes equation (2) in velocity-vorticity formulation. The term $\left(\boldsymbol{\Omega}_{i} \cdot \boldsymbol{\nabla}\right) \mathbf{U}_{i}$, only in 3D configurations, is the deformation term and deals 
with the stretching and reorientation of the vortical particles in the flow (see $[29,27])$.

A second order Runge-Kutta method is used as the time integration scheme for equations (21) and (22) in order to have a good compromise between accuracy and time saving. To prevent the calculation from collapsing, a global re-meshing of the particles is also included every four time steps. This re-meshing process uses the interpolation formula $M_{4}^{\prime}$. A more complete survey on the effect of global re-meshing is available in [27], where different interpolation formulae have been studied in terms of the velocity $L_{2}$ error introduced in the simulation. These studies concluded that the third order interpolation formula $M_{4}^{\prime}$ provides a good compromise between accuracy, smoothness of the results and implementation work. The present computations are run on parallel architectures thanks to MPI (Message Passing Interface) libraries.

\section{Viscous and turbulent diffusion: PSE scheme and LES model}

In this section, the discretisation of the diffusion term of the Navier-Stokes equation (22) will be discussed. In the present numerical tool, a turbulence model based on a simple LES (Large Eddy Simulation) is implemented. For this reason, non uniform viscosity has to be considered. A more general way to treat diffusion with non uniform viscosity would be to use the term $\boldsymbol{\nabla} \cdot(\nu \boldsymbol{\nabla} \boldsymbol{\omega})$. It is precisely this term that is discretised in the following paragraphs. And in the case of homogeneous and constant viscosity, this last term $\boldsymbol{\nabla} \cdot(\nu \boldsymbol{\nabla} \boldsymbol{\omega})$ basically is reduced to $\nu \Delta \boldsymbol{\omega}$.

The Particle Strength Exchange (PSE) method [30, 31, 32], as its name points out, models diffusion with a transfer of vorticity from one particle to another without changing the support $\mathcal{P}_{i}$ of the blobs. The following equations lead to the discrete formulation. First of all, an expression for the vorticity is defined in equation (23):

$$
\boldsymbol{\omega}(\mathbf{x})=\int_{\mathcal{V}} \boldsymbol{\omega}\left(\mathbf{x}^{\prime}\right) \zeta_{\varepsilon}\left(\left|\mathbf{x}-\mathbf{x}^{\prime}\right|\right) d \mathbf{x}^{\prime}
$$

with $\varepsilon$ the cut-off parameter, and $\zeta_{\varepsilon}$ a 3D-radially symmetric regular function of unit weight, whose limit, as $\varepsilon \rightarrow 0$, is the Dirac measure. The following smoothing function is chosen:

$$
\zeta_{\varepsilon}(r)=\frac{1}{\left(\pi \varepsilon^{2}\right)^{3 / 2}} \exp \left(-\frac{r^{2}}{\varepsilon^{2}}\right) .
$$

The diffusion term $\boldsymbol{\nabla} \cdot(\nu \boldsymbol{\nabla} \boldsymbol{\omega})$ with non uniform viscosity $\nu$ is then evaluated as in equation (25), defining a function $\eta_{\varepsilon}(r)$ calculated from $\zeta_{\varepsilon}(r)$ (cf. [31]). The expression for the diffusive part of the Navier-Stokes equation (22) is now obtained by an integral representation.

$$
\begin{aligned}
\boldsymbol{\nabla} \cdot(\nu \boldsymbol{\nabla} \boldsymbol{\omega})(\mathbf{x})=\int_{\mathcal{V}}\left(\nu(\mathbf{x})+\nu\left(\mathbf{x}^{\prime}\right)\right)\left(\boldsymbol{\omega}\left(\mathbf{x}^{\prime}\right)-\boldsymbol{\omega}(\mathbf{x})\right) \eta_{\varepsilon}\left(\left|\mathbf{x}-\mathbf{x}^{\prime}\right|\right) d \mathbf{x}^{\prime} \\
\text { with } \eta_{\varepsilon}(r)=-\frac{\boldsymbol{\nabla} \zeta_{\varepsilon} \cdot \mathbf{r}}{r^{2}}=\frac{2}{\varepsilon^{2}} \zeta_{\varepsilon}(r)
\end{aligned}
$$

The next step consists in discretising this last equation (25): 


$$
(\boldsymbol{\nabla} \cdot(\nu \boldsymbol{\nabla} \boldsymbol{\omega}))_{\left(\mathbf{x}=\mathbf{X}_{i}\right)} \mathcal{V}_{i} \approx \sum_{j=1}^{N_{\Omega}}\left(\nu_{i}+\nu_{j}\right)\left(\boldsymbol{\Omega}_{j} \mathcal{V}_{i}-\boldsymbol{\Omega}_{i} \mathcal{V}_{j}\right) \eta_{\varepsilon}\left(\left|\mathbf{X}_{i}-\mathbf{X}_{j}\right|\right)
$$

with $\mathcal{V}_{i}$ the volume of the $i^{\text {th }}$ particle.

Equation (26) completes the discretisation of the diffusive part of the Navier Stokes equations. As mentioned above, a simple turbulence model is added on top of the molecular viscous diffusion. This is an LES model based on enstrophy introduced by Mansour et al. [33], defining an eddy viscosity $\nu_{i}^{t u r b}$. The total viscosity $\nu_{i}$ used in equation (26) is the sum of the molecular viscous term and the turbulent term, $\nu_{i}=\nu^{\text {visc }}+\nu_{i}^{\text {turb }}$ with:

$$
\nu_{i}^{\text {turb }}=\left(C_{v} h\right)^{2} \sqrt{2}\left|\boldsymbol{\omega}\left(\mathbf{X}_{i}\right)\right|
$$

Some mathematics can be found in [34] in order to demonstrate the previous form $\nu_{i}^{\text {turb }}$ (named alternate form in [34], p. 115) from the Kolmogorov hypothesis of isotropic homogeneous turbulence. The limitations of the current model mainly reside in the fact that the hypothesis of isotropic homogeneous turbulence is not met in the present application. An improved model, using a dynamic LES scheme can be found in Mansfield et al. [35]; one can also refer to [36] for a review. The present computations use a somewhat simpler version, particularly with respect to the value of the constant $C_{v}$, which is fixed a priori and set to a typical value of 0.15 . The length $h$ is equal to the smoothing parameter $\varepsilon$, which is an intuitive and widely spread way of determining the sub-grid scale. The motivations for choosing this model are twofold. Firstly, it is quite easy to implement within a particle method and secondly, it is built for vorticity diffusion, which is exactly what is required. It should be noted that in these computations, the total viscosity $\nu_{i}$ is very close to the eddy viscosity $\nu_{i}^{\text {turb }}$, since $\nu^{\text {visc }}$ turns out to be small compared to $\nu_{i}^{\text {turb }}$. This kind of behaviour is generally true except for moderate or low Reynolds numbers. The concerned Reynolds numbers are presented in Table 1 and are discussed hereafter.

\subsection{Vortex particles emission at the trailing edge}

This section is mainly inspired from [37] and [38]. The vortex particles emission at the trailing edge uses the unsteady Bernoulli relation around the lifting surface which is, in the present case, the blades. The blades are considered here in the thin air-foil theory, i.e. the blade thickness is not taken into consideration as it is supposed to be infinitely thin. The boundary layers are not computed either, which implies that cases of flow separation cannot be treated in the present implementation of the numerical tool.

Denoting by $\left(^{+}\right)$the upper face of the lifting surface (extrados) and $\left(^{-}\right)$the lower face (intrados), the Bernoulli relation may be written

$$
\frac{\partial\left(\phi^{+}-\phi^{-}\right)}{\partial t}+\frac{\left(p^{+}-p^{-}\right)}{\rho}+\frac{1}{2}\left(\mathbf{u}^{+^{2}}-\mathbf{u}^{-2}\right)=0 .
$$

At the trailing edge, there is $p^{+}=p^{-}$and the previous equation (28) becomes

$$
\frac{\partial\left(\phi^{+}-\phi^{-}\right)}{\partial t}+\frac{\left(\mathbf{u}^{+}+\mathbf{u}^{-}\right)}{2}\left(\mathbf{u}^{+}-\mathbf{u}^{-}\right)=0
$$


With $\mu$ representing the potential jump $\left(\phi^{+}-\phi^{-}\right)$through the lifting surface, the following definitions are obtained:

$$
\nabla \mu=\nabla\left(\phi^{+}-\phi^{-}\right)=\mathbf{u}^{+}-\mathbf{u}^{-},
$$

and denoting by $\mathbf{u}_{m}$ the mean velocity

$$
\mathbf{u}_{m}=\frac{1}{2}\left(\mathbf{u}^{+}+\mathbf{u}^{-}\right)
$$

we finally obtain from equation (29) that the normal dipoles distribution $\mu$ is advected by the mean flow at the trailing edge:

$$
\frac{\partial \mu}{\partial t}+\mathbf{u}_{m} \cdot \nabla \mu=\frac{D \mu}{D t}=0
$$

The normal dipole, or somehow the vortex sheet, is thus introduced in the flow field at the trailing edge of the blade with the velocity $\mathbf{u}_{m}$.

On a lifting surface, a bound vortex layer is attached to the surface. The relation between the normal dipole distribution $\mu$ and the bound vortex strength $\gamma$ is the following:

$$
\gamma=\mathbf{n} \wedge \nabla \mu
$$

with $\mathbf{n}$ the vector normal to the considered surface. Looking at the discretised form on the $k$-surface element discretising the blade, we have

$$
\gamma_{k}=\mathbf{n}_{k} \wedge \nabla \mu_{k}
$$

and the integrated form to obtain an equivalent to a bound vortex particles attached to the blade is

$$
\Omega_{k}=\iint_{\mathcal{S}_{k}} \gamma_{k} d s_{k}
$$

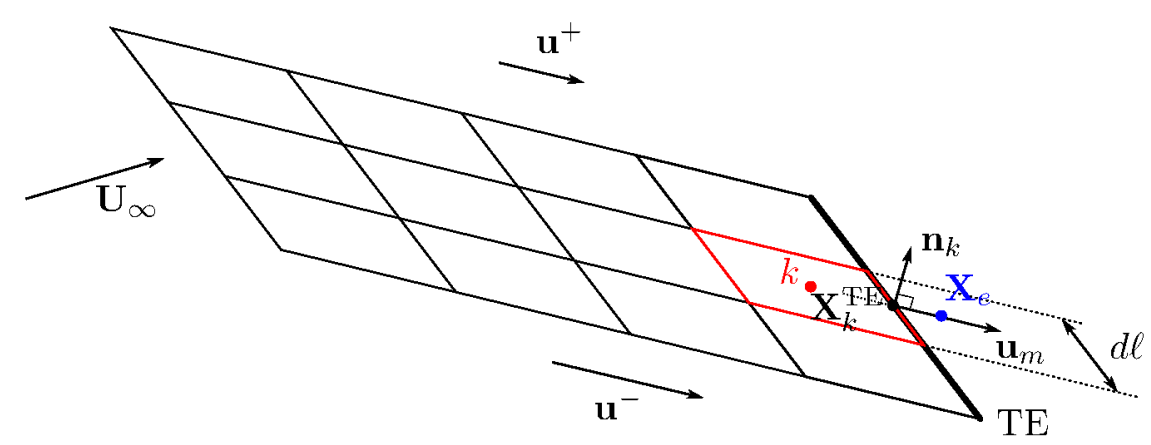

Figure 2: Schematic representation of a particle emission at the trailing edge

At the trailing edge, as equation (32) and Figure 2 explain, the bound vortex sheet is advected in the flow with velocity $\mathbf{u}_{m}$, so the emitted particle $\left(\mathbf{X}_{e}, \boldsymbol{\Omega}_{e}\right)$ can be expressed as follows:

$$
\begin{aligned}
\boldsymbol{\Omega}_{e} & =\int_{d t} \int_{d \ell}\left|\mathbf{u}_{m}\right| \boldsymbol{\gamma}_{T E} \delta t \delta \ell \\
\mathbf{X}_{e} & =\mathbf{X}_{T E}+\frac{1}{2} \mathbf{u}_{m} d t
\end{aligned}
$$


with $\gamma_{T E}$ obtained with equation (34) and the approximation $\left|\mathbf{u}_{m}\right| d t d \ell \approx d s_{e}$. Hence, equation (36) can be rewritten as:

$$
\boldsymbol{\Omega}_{e}=\int_{d t} \int_{d \ell}\left|\mathbf{u}_{m}\right|\left(-\frac{\partial \mu}{\partial x_{j}} \mathbf{i}+\frac{\partial \mu}{\partial x_{i}} \mathbf{j}\right) \delta t \delta \ell,
$$

with $\mathbf{i}$ a unit vector aligned with the trailing edge and $\mathbf{j}$ defined so that $\left(\mathbf{i}, \mathbf{j}, \mathbf{n}_{k}\right)$ is a local orthonormal direct vector base. The partial derivative $\frac{\partial \mu}{\partial x_{i}}$ can easily be obtained by a simple finite difference:

$$
\boldsymbol{\Omega}_{e} \cdot \mathbf{j}=\int_{d t} \int_{d \ell}\left|\mathbf{u}_{m}\right| \frac{\partial \mu}{\partial x_{i}} \delta t \delta \ell=d t\left|\mathbf{u}_{m}\right| \frac{\mu_{k+1}-\mu_{k-1}}{2},
$$

where the vortex sheet strength is assumed to be zero on the outer lateral border of the blade. Evaluating the second derivative $-\frac{\partial \mu}{\partial x_{j}}$ is a little more complicated and equation (32) should be recalled. From this equation, it must be remembered that the attached vortex sheet is advected at the trailing edge by $\mathbf{u}_{m}$ and can be written

$$
\frac{\partial \mu}{\partial x_{j}}=\frac{-1}{\left(\mathbf{u}_{m} \cdot \mathbf{j}\right)}\left(\frac{\partial \mu}{\partial t}+\left(\mathbf{u}_{m} \cdot \mathbf{i}\right) \frac{\partial \mu}{\partial x_{i}}\right) \approx \frac{-1}{\left|\mathbf{u}_{m}\right|} \frac{\partial \mu}{\partial t},
$$

and the last component of $\Omega_{e}$ is obtained under the following form

$$
\boldsymbol{\Omega}_{e} \cdot \mathbf{i}=\int_{d t} \int_{d \ell} \frac{\partial \mu}{\partial t} \delta t \delta \ell=d \ell\left(\mu_{k}(t+d t)-\mu_{k}(t)\right) .
$$

Several conclusions can be drawn from these equations. More particularly, from equation (41), we can say that the unsteady contribution of the vortex sheet is mainly aligned with the trailing edge (i). However, the three-dimensional effects are perpendicular to the trailing edge normal $(\mathbf{j})$, which is obvious from equation (39) and consistent with the fact that these three-dimensional effects appear only if there is a variation along the span direction. Details of the previous demonstration can be found in [37].

\subsection{Elementary force evaluation}

As the bound vortex sheet $\gamma$, which is linked to the normal dipole distribution $\mu$, has been evaluated in the rotating frame $\mathcal{R}_{\text {rot }}$, an additional pseudo Coriolis force $\mathbf{f}_{c}=-2 \Phi \wedge \mathbf{U}$ has to be considered. For any lifting surface in an unsteady flow, the total force $\mathcal{F}$ may be evaluated as follows:

$$
\mathcal{F}=\iint_{\mathcal{S}} \rho \frac{\partial \mu}{\partial t} d s+\iint_{\mathcal{S}} \rho \mathbf{U} \wedge \gamma d s+\iint_{\mathcal{S}}-2 \rho \mathbf{\Phi} \wedge \mathbf{U} d s .
$$

A discretised form of this equation for an elementary panel of surface $s_{k}$ and vector normal $\mathbf{n}_{k}$ would be the following:

$$
\mathbf{f}_{k}=\rho \frac{\mu_{k}(t+d t)-\mu_{k}(t)}{\delta t} s_{k} \mathbf{n}_{k}+\sum_{n=1}^{4} \rho v_{n} \mu_{k} \mathbf{U}\left(P_{n, k}\right) \wedge \mathbf{d l}_{n, k}-2 \rho \mathbf{\Phi} \wedge \mathbf{U}\left(P_{k}\right) s_{k}
$$

with the following definitions: 
- $\mathbf{U}\left(P_{n, k}\right)$ the velocity at the centre of the $n^{\text {th }}$ segment of the $k^{\text {th }}$ elementary panel,

- $\mathbf{d l}_{n, k}$ the vector corresponding to this $n^{\text {th }}$ segment of the $k^{\text {th }}$ elementary panel,

- $v_{n}$ a coefficient equal to the surface ratio between the two neighbouring elements, if the $n^{\text {th }}$ segment belongs to two elementary panels, and 1 otherwise,

- $\mathbf{U}\left(P_{k}\right)$ the velocity at the centre of the $k^{\text {th }}$ elementary panel.

With these definitions, the total force $\mathcal{F}$ and moment $\mathcal{M}_{O}$ at the centre of rotation (i.e. the frame centre $O$ ) can be defined as follows:

$$
\begin{aligned}
\mathcal{F} & =\sum_{k=1}^{N_{f}} \mathbf{f}_{k} \\
\mathcal{M}_{O} & =\sum_{k=1}^{N_{f}} \mathrm{OP}_{k} \wedge \mathbf{f}_{k}
\end{aligned}
$$

\section{Turbine description and discretisation}

\subsection{Marine current turbine configurations}

In order to validate the numerical tool, two different three bladed horizontal axis turbine configurations were used, both experimentally tested in flume tanks (cf. Table 1). The first set of blades is composed of NACA 63-8xx profiles. They were experimentally and numerically tested first by Bahaj et al. [1] and by Batten et al. [14]. For this reason, this set of blades will be named hereafter BBMC for Bahaj Batten McCann. A description of chord $c$, thickness $t$ and pitch distribution is given in Table 2. In [1], a large number of results can be found with four different set angles, $0^{\circ}, 5^{\circ}, 10^{\circ}$ and $13^{\circ}$. The second set of blades used for validation purposes was first designed by Tidal Generation Limited (TGL) [39] and experimentally tested in the IFREMER flume tank of Boulogne-sur-Mer (France) [2]. However, this set of blades, presented in Maganga et al. [2], is patented so a similar and open set of blades was developed for experimental validation at IFREMER and numerical trial at LOMC-Le Havre University (France). In the present paper, this last open blades configuration will be named as the IFREMER-LOMC configuration. The turbine blades are designed from a NACA63418 profile. More detailed descriptions can be found in Table 2 .

The Tip Speed Ratio $(T S R)$ is defined as:

$$
T S R=\frac{\Phi R}{U^{\infty}}
$$

where $R$ stands for the turbine radius (cf. Table 1), $\Phi$ for the rotational speed and $U^{\infty}$ represents the modulus of the upstream current velocity, which is supposed to be a constant vector in the present study. The Reynolds number $\left(\operatorname{Re}_{\infty}\right)$ based 
on the rotor radius $R$, the upstream velocity $U^{\infty}$ and the kinematic viscosity at $20^{\circ} \mathrm{C}$, is given for both configurations in Table 1 . All the computations were run dimensionless with a rotor radius $R=1.0$ and $U^{\infty}=1.0$. So depending on the tested configuration, the numerical molecular viscosity $\nu_{v i s c}$ was adjusted in order to obtain Reynolds' invariance. In both cases, BBMC and IFREMER-LOMC configurations, $\nu_{\text {visc }}$ were then very small, which corroborates with the previous remark that the numerical molecular viscosity was very small if compared to the eddy viscosity $\nu_{\text {turb }}$ (see section 2.2).

\begin{tabular}{l|c|c} 
Description & BBMC [1] & IFREMER-LOMC \\
\hline Rotor Radius $(R)$ & $400 \mathrm{~mm}$ & $350 \mathrm{~mm}$ \\
Hub Radius & $50 \mathrm{~mm}$ & $46 \mathrm{~mm}$ \\
Hub length & $\mathrm{n} / \mathrm{a}$ & $720 \mathrm{~mm}$ \\
Pitch (set angle) & $0^{\circ}, 5^{\circ}, 10^{\circ}$ and $13^{\circ}$ & $0^{\circ}$ \\
TSR & {$[2-10]$} & {$[0-10]$} \\
Sense of rotation & clockwise & anti-clockwise \\
Reynolds $\left(\operatorname{Re}_{\infty}\right)$ & $\approx 580,000$ & $\approx 280,000$
\end{tabular}

Table 1: Marine current turbine general description for both $B B M C$ [1] and IFREMER-LOMC configurations.

Several observations can be drawn from blades descriptions (Table 1 and 2). Firstly, the rotation sense is clockwise for the $B B M C$ configuration and anti-clockwise in the present configuration owing to the IFREMER-LOMC experimental model. Apart from that, the general configuration of both models is very similar in rotor and hub size as well as in tested TSR values. However, the blades' configurations are quite different: the dimensionless chord size $c / R$ of the IFREMER-LOMC blades is larger than that of the $B B M C$ blades, which are slimmer and more elongated. In the $[0.2-1.0] r / R$ region, the blades' thickness $t$ is almost always inferior to $25 \%$ of the chord $c$ in both cases. However, owing to the lifting surface theory, the blades' thickness will not be considered in the present numerical study as the lifting surface is supposed to be infinitely thin. Another important aspect resides in the fact that the present IFREMER-LOMC configuration is more twisted with a total angle variation of approximately $20^{\circ}$ compared to less than $15^{\circ}$ in the $B B M C$ case. The three latter observations might be important issues for the possible explanation of a slight discrepancy in the results (see section 4.2). The Galilean reference frame $\mathcal{R}_{g}$ is presented in Figure 3.

\subsection{Blade mesh description}

In order to proceed to the convergence analysis of the numerical model, different surface meshes of the three-bladed marine current turbines were designed including the meshing of the hub. Geometrical characteristics are described in Figure 4, where notations are given for the blades' discretisation (cf. Table 3). The convergence analysis, presented in section 4.1, was performed considering the smoothing parameter $\varepsilon$, which is proportional to the inter-particle spacing $d h$. Here, we take:

$$
\varepsilon=1.5 \times d h .
$$




\begin{tabular}{|c|c|c|c|c|c|c|c|}
\hline & & & & $r / R$ & $c / R$ & Pitch (deg) & $t / c(\%)$ \\
\hline & & & & 0.13 & 0.06 & 29.57 & 80 \\
\hline & & & & 0.15 & 0.06 & 29.57 & 100 \\
\hline & & & & 0.16 & 0.06 & 29.57 & 100 \\
\hline & & & & 0.20 & 0.15 & 25.63 & 36 \\
\hline & & & & 0.24 & 0.25 & 22.15 & 21 \\
\hline & & & & 0.29 & 0.24 & 19.30 & 21 \\
\hline$r / R$ & $c / R$ & Pitch (deg) & $t / c(\%)$ & 0.33 & 0.23 & 16.97 & 22 \\
\hline 0.20 & 0.125 & 15.0 & 24.0 & 0.37 & 0.21 & 15.05 & 22 \\
\hline 0.30 & 0.116 & 9.5 & 20.7 & 0.42 & 0.20 & 13.46 & 22 \\
\hline 0.40 & 0.106 & 6.1 & 18.7 & 0.46 & 0.19 & 12.12 & 22 \\
\hline 0.50 & 0.097 & 3.9 & 17.6 & 0.50 & 0.18 & 10.98 & 23 \\
\hline 0.60 & 0.088 & 2.4 & 16.6 & 0.55 & 0.17 & 10.01 & 23 \\
\hline 0.70 & 0.078 & 1.5 & 15.6 & 0.59 & 0.17 & 9.18 & 22 \\
\hline 0.80 & 0.069 & 0.9 & 14.6 & 0.63 & 0.16 & 8.45 & 22 \\
\hline 0.90 & 0.059 & 0.4 & 13.6 & 0.68 & 0.15 & 7.82 & 22 \\
\hline 1.00 & 0.050 & 0.0 & 12.6 & 0.72 & 0.15 & 7.26 & 21 \\
\hline & & (a) BBMC & & 0.76 & 0.14 & 6.77 & 21 \\
\hline & & & & 0.81 & 0.14 & 6.34 & 20 \\
\hline & & & & 0.85 & 0.13 & 5.95 & 19 \\
\hline & & & & 0.89 & 0.13 & 5.61 & 19 \\
\hline & & & & 0.94 & 0.12 & 5.29 & 18 \\
\hline & & & & 0.98 & 0.12 & 5.01 & 18 \\
\hline & & & & 1.00 & 0.07 & 4.87 & 25 \\
\hline
\end{tabular}

(b) IFREMER-LOMC

Table 2: Detailed blade geometry description of the $B B M C$ configuration reproduced from Bahaj et al. [1] (left) and the present IFREMER-LOMC configuration (right).

In Lagrangian methods, there is no Eulerian meshing of the fluid domain, and only vortical zones are represented by vorticity-carrying particles $\left(\mathbf{X}_{i}, \boldsymbol{\Omega}_{i}\right)$ (see section 2.2).

For a given smoothing parameter $\varepsilon$ (or equivalently for a given $d h$ ), a corresponding $l_{T E}$ equal to $d h$ is chosen to have a characteristic mesh size at the trailing edge of the blades (cf. Figure 4). Section 2.3 can be referred to for more explanation about $d h=l_{T E}$. In addition, different chord discretisations were tested, corresponding to different values of $l_{c}$, as shown in figure 4 . The three tested chord discretisations are namely $N_{c}=5,10$ and 15 . For a given smoothing parameter $\varepsilon$ (equivalently $d h$ ), the time step $d t$ is obtained according to the turbine rotational speed $\Phi$, in order to preserve the following condition for each particle:

$$
d h \approx|\mathbf{U}| d t
$$

which can be seen as a Lagrangian frame equivalent to the $C F L$ condition in Eulerian computations. For the most critical case, i.e. the particle emitted at the top end of 


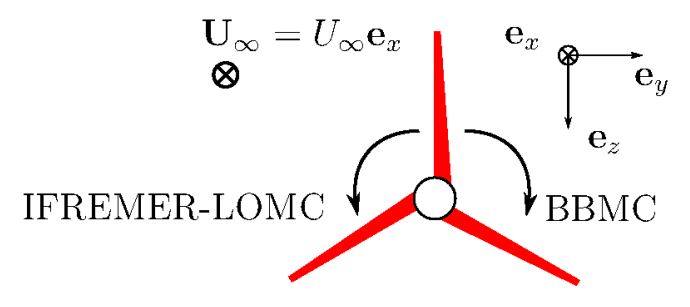

Figure 3: Reference frame for the computations in both configurations

\begin{tabular}{cccccc}
$\varepsilon$ & $\begin{array}{c}d h \\
(\varepsilon / 1.5)\end{array}$ & $\begin{array}{c}N_{T E} \\
(\text { BBMC) }\end{array}$ & $\begin{array}{c}N_{T E} \\
(\text { IFREMER-LOMC })\end{array}$ & $\begin{array}{c}\min d t \\
(T S R=0)\end{array}$ & $\begin{array}{c}\max d t \\
(T S R=10)\end{array}$ \\
\hline 0.200 & 0.133 & 6 & 5 & 0.133 & 0.013 \\
0.150 & 0.100 & 8 & 7 & 0.100 & 0.009 \\
0.100 & 0.067 & 12 & 11 & 0.066 & 0.006 \\
0.075 & 0.050 & 16 & 15 & 0.050 & 0.004 \\
0.050 & 0.033 & 24 & 23 & 0.033 & 0.003
\end{tabular}

Table 3: Mesh description for different values of $\varepsilon$

the blade, the following relation for the determination of $d t$ is finally obtained:

$$
d t \leq \frac{d h}{\sqrt{\Phi^{2} R^{2}+U_{\infty}^{2}}}=\frac{\varepsilon}{1.5 U_{\infty} \sqrt{(T S R)^{2}+1}} .
$$

For each combined $\varepsilon$ and $T S R$ values, a value for $d t$ is chosen according to equation (49) and the time step is fixed initially for the whole unsteady computation (see Table 3).

Figure 5 depicts raw results of the computations with particles coloured according to their vortical intensity $\left|\Omega_{i}\right|$ and the IFREMER-LOMC turbine coloured by dimensionless pressure jump. Mainly two types of computations were run in order to evaluate either the power and thrust coefficients or the wake characterisation. In order to evaluate the power and thrust coefficients, the computations were run over a total elapsed time of $\Delta t_{\text {tot }}=3 \mathrm{~s}$ real time in order to have stable values of rotational torques and forces. The results are presented in section 4 . In order to evaluate wake quantities (velocity profiles, axial velocity decay, etc.) in the far field (10 diameters downstream), longer computations were run until $t=30 \mathrm{~s}$ real time with approximately one million particles and longer CPU durations (cf. section 5).

\section{Power coefficient evaluation}

From raw results depicted in Figure 5, the total force $\mathcal{F}$ and moment $\mathcal{M}$ applied to the turbine at each time step can be computed according to equations (44) and (45) presented in section 2.4. In order to avoid the transient effect at the beginning of the computations (for $t \leq 1 \mathrm{~s}$ in Figure 6 ), mean values of the force $\mathcal{F}$ and moment $\mathcal{M}$ are computed, as well as standard deviation, for an elapsed time between 1 and $3 \mathrm{~s}$. The Power Coefficient $C_{P}$ and Thrust Coefficient $C_{T}$ for a marine current turbine 


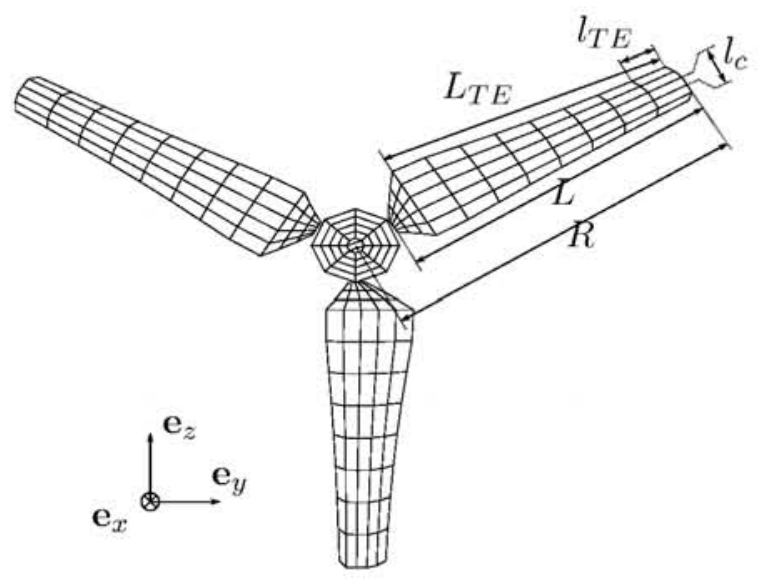

Figure 4: Description of the meshing parameters on a IFREMER-LOMC model

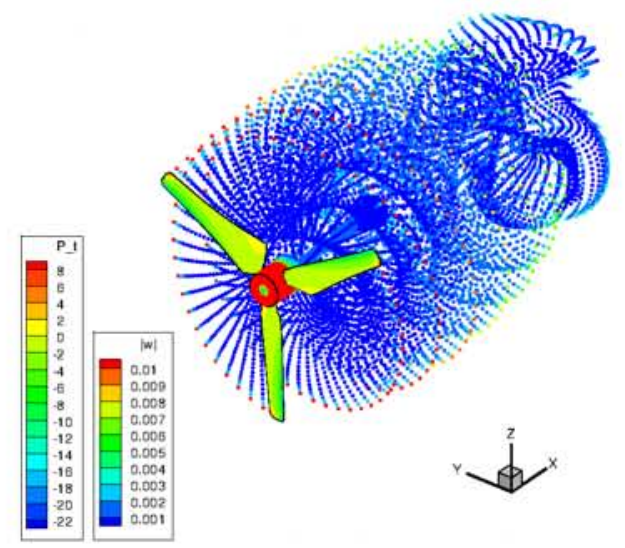

Figure 5: Particles coloured by vortical intensity $\left|\Omega_{i}\right|$ and the IFREMER-LOMC turbine coloured by dimensionless pressure jump through the surface.

are defined as:

$$
C_{P}=\frac{\mathcal{M}_{x} \Phi}{0.5 \rho\left|\mathbf{U}^{\infty}\right|^{3} \pi R^{2}}=\frac{\mathcal{M}_{x}(T S R)}{0.5 \rho\left|\mathbf{U}^{\infty}\right|^{2} \pi R^{3}} \quad \text { and } \quad C_{T}=\frac{\mathcal{F}_{x}}{0.5 \rho\left|\mathbf{U}^{\infty}\right|^{2} \pi R^{2}},
$$

with $\mathcal{M}_{x}$ the moment around the x-coordinate (i.e. the turbine torque), $\mathcal{F}_{x}$ the total force along the x-coordinate and $\Phi$ the scalar rotational speed. Graphs of the Power Coefficient $C_{P}$ and Thrust Coefficient $C_{T}$ are presented in the following section as a function of $T S R$, the Tip Speed Ratio (eq. (46)). In the near future, tests will be run in order to deal with different upstream conditions, for instance a sea boundary layer profile [17] or combined wave and current velocity profiles [40].

As a matter of validation of the numerical tool, a convergence analysis was performed for different computation resolutions depending on the $\varepsilon$ parameter as presented in Table 3. A complete description of the computations can be found in Table 3 and Figure 4 including blade's mesh resolution, inter-particles spacing, number of emitted particles and time steps. Figures 7 to 10 present the convergence analysis for different parameters and for the two tested configurations. Figures 11 and 12 compare the converged results with experimental and other numerical results in literature. 


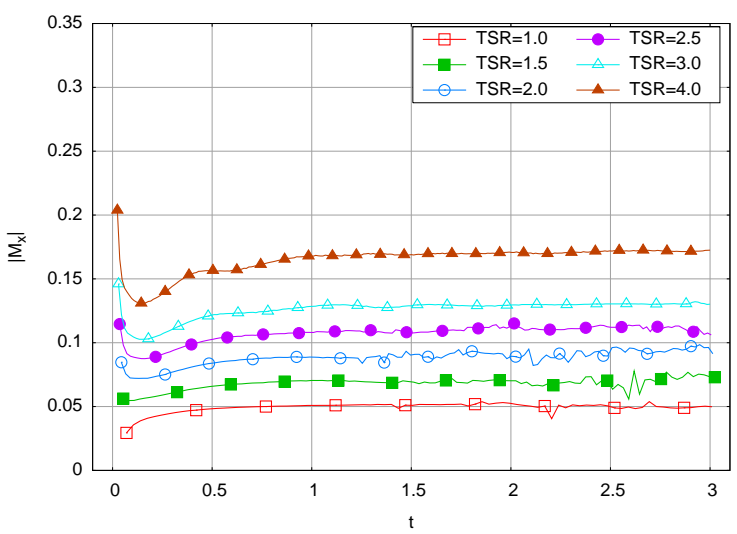

(a) $B B M C$ configuration with a $5^{\circ}$ set angle

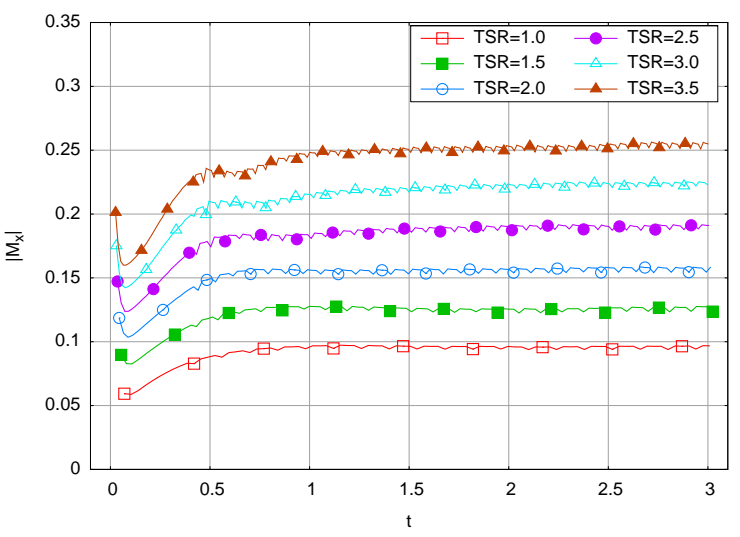

(b) IFREMER-LOMC configuration

Figure 6: Torque around the x-coordinate $\left(\mathcal{M}_{x}\right)$ as a function of time, for different TSR with $N_{c}=5$ and $\varepsilon=0.075$ for both configurations.

\subsection{Convergence analysis}

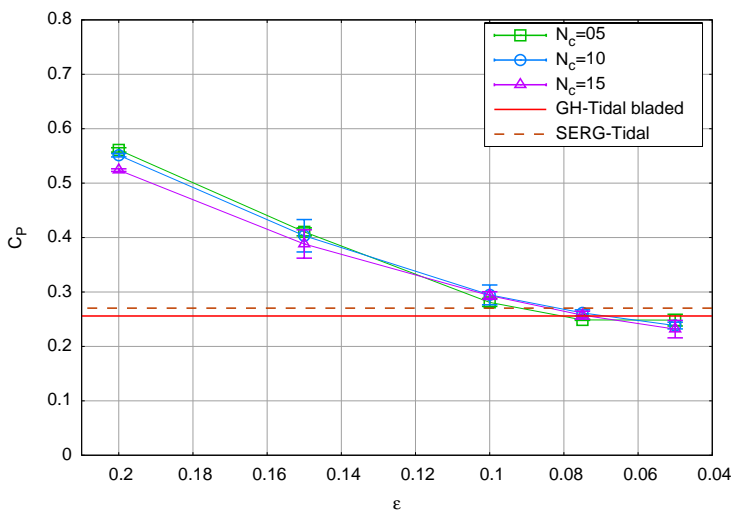

(a) $C_{P}$

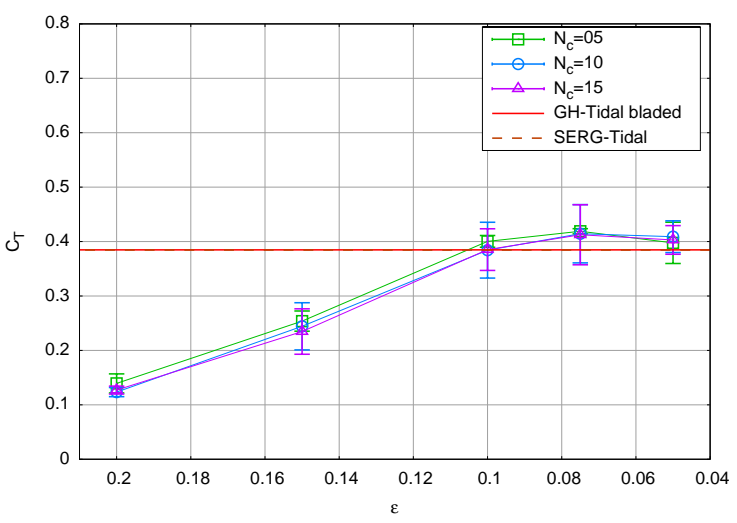

(b) $C_{T}$

Figure 7: Convergence analysis of $C_{P}$ and $C_{T}$ values on the $B B M C$ configuration, with a $5^{\circ}$ set angle and $T S R=3$, function of $\varepsilon$ and thus of the corresponding trailing edge discretisation $N_{T E}$ and inter-particle spacing $d h$ (cf. Table 3). Results are given for different chord discretisations $N_{c}$. Mean values are presented with symbols and standard deviations as error bars. The GH-Tidal bladed and SERG-Tidal results were reproduced from Bahaj et al. [1].

From Figure 7, it can be observed that the computations converge to a coherent value either on $C_{P}$ or $C_{T}$ compared to GH-Tidal bladed and SERG-Tidal results reproduced from Bahaj et al. [1]. Mean values are presented with symbols and standard deviation with error bars. From these results it can be concluded that the computations are converged, at first order, from $\varepsilon \lesssim 0.1$. From these graphs, three observations can be drawn: first, for large $\varepsilon$ values, $C_{P}$ and $C_{T}$ evaluation errors can be very important. Convergence is then obtained more rapidly in terms of $C_{T}$ than for $C_{P}$. Finally, at first order, it seems that the chord discretisation only has a minor influence on the results. From Figure 8, where only a single chord 


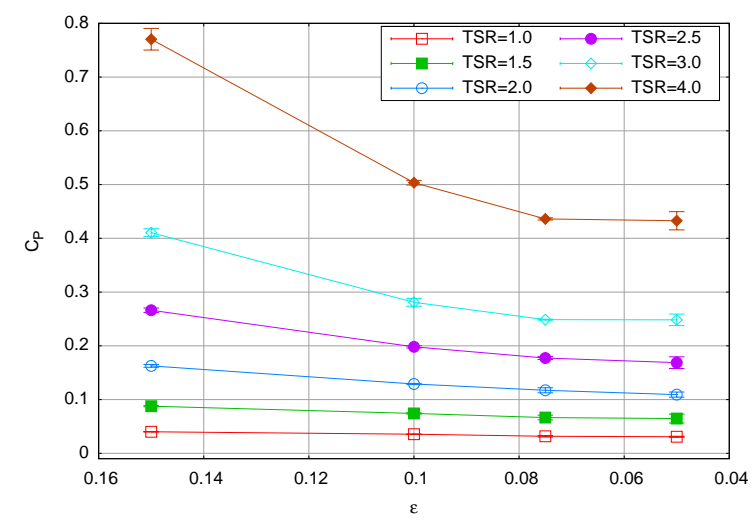

(a) $C_{P}$

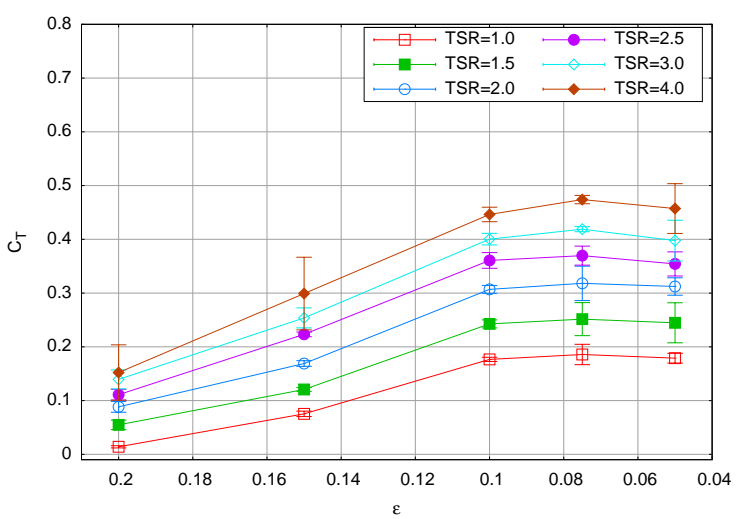

(b) $C_{T}$

Figure 8: Convergence analysis of $C_{P}$ and $C_{T}$ values on the $B B M C$ configuration, with a $5^{\circ}$ set angle and a fixed chord discretisation $N_{c}=5$, function of $\varepsilon$ and thus of the corresponding trailing edge discretisation $N_{T E}$ and inter-particle spacing $d h$ (cf. Table 3). Results are given for different $T S R$ values. Mean values are presented with symbols and standard deviations as error bars.

discretisation $N_{c}=5$ is depicted, it can be seen that convergence is obtained for each $T S R$ value from $\varepsilon \lesssim 0.1$.

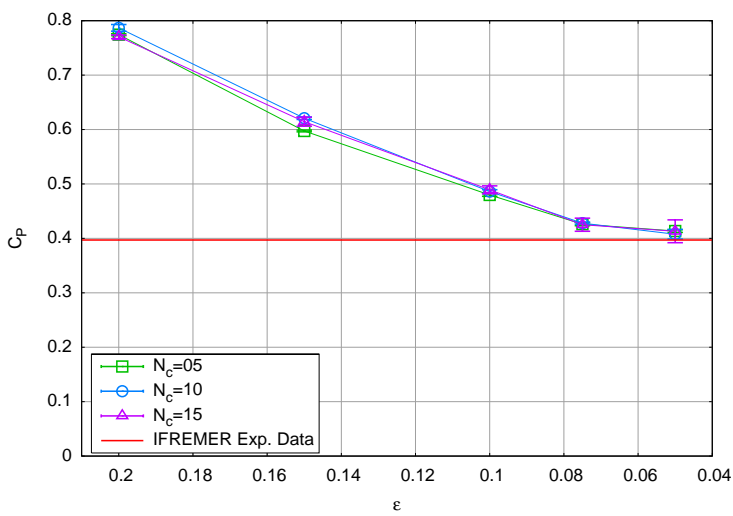

(a) $C_{P}$

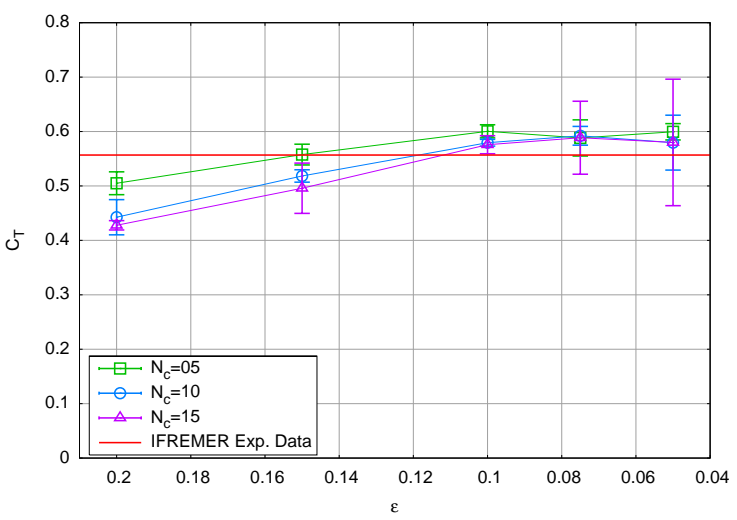

(b) $C_{T}$

Figure 9: Convergence analysis of $C_{P}$ and $C_{T}$ values on the IFREMER-LOMC configuration with $T S R=3$ function of $\varepsilon$ and thus of the corresponding trailing edge discretisation $N_{T E}$ and inter-particle spacing $d h$ (cf. Table 3). Results are given for different chord discretisations $N_{c}$. Mean values are presented with symbols and standard deviations as error bars. The experimental results were obtained in the IFREMER flume tank in Boulogne-sur-Mer (France); the experimental procedure is presented in Maganga et al. [2].

Figures 9 and 10 present the same parametric study for the IFREMER-LOMC turbine configuration (cf. Tables 1 and 2). Comparing Figures 7 and 9 in terms of $C_{P}$, it can be observed that the convergence is still obtained but for lower $\varepsilon$ values (i.e. $\varepsilon \lesssim 0.075)$ than for the $B B M C$ configuration $(\varepsilon \lesssim 0.1)$. On the contrary, the 


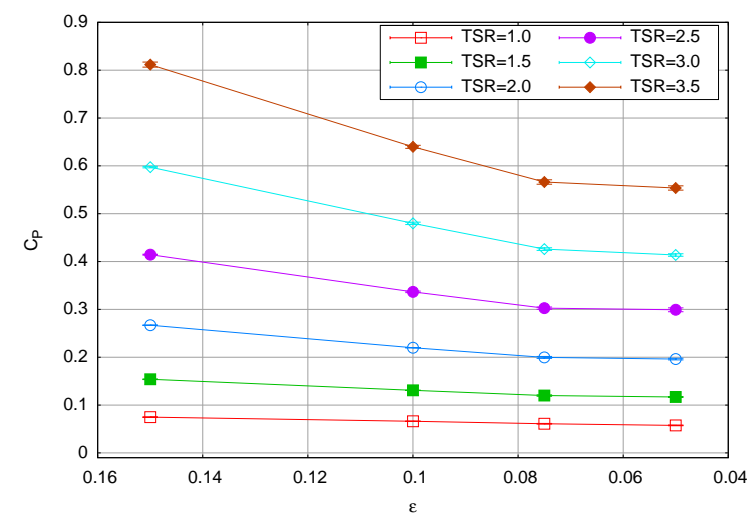

(a) $C_{P}$

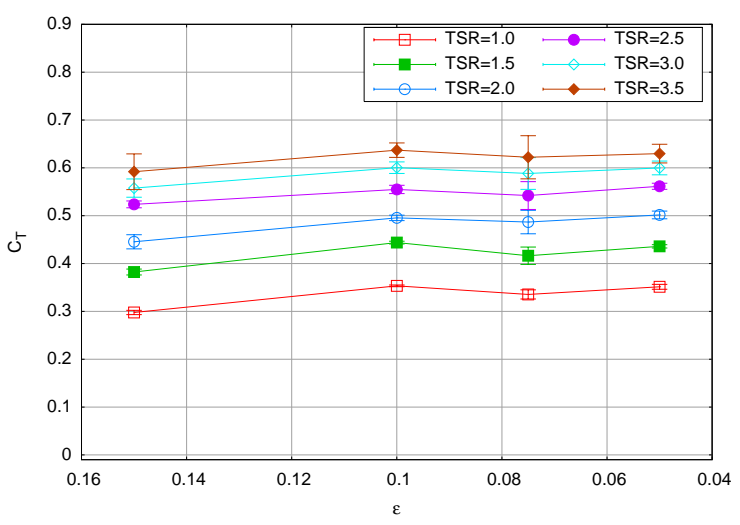

(b) $C_{T}$

Figure 10: Convergence analysis of $C_{P}$ and $C_{T}$ values on the IFREMER-LOMC configuration, with a fixed chord discretisation $N_{c}=5$, function of $\varepsilon$ and thus of the corresponding trailing edge discretisation $N_{T E}$ and inter-particle spacing $d h$ (cf. Table 3). Results are given for different $T S R$ values. Mean values are presented with symbols and standard deviations as error bars.

$C_{T}$ convergence seems to be easily obtained with the IFREMER-LOMC configuration. In the authors' opinion, this can be interpreted by the fact that the blades are significantly more twisted in the IFREMER-LOMC geometry than they are in the $B B M C$ one. Other geometrical aspects as the dimensionless blade chord $c / R$ and relative thickness $t / c$ might be of importance as well (cf. Table 2).

It is important to recall now that the aim of the present study is twofold: Power Coefficient $C_{P}$ and Thrust Coefficient $C_{T}$ evaluation on the one hand as well marine current turbine wake characterisation on the other hand. Further convergence in $C_{P}$ and $C_{T}$, together with lower $\varepsilon$ values is possible, but it would lead to such an considerable increase in the number of particles that the computations would be inaccessible in terms of CPU resources (see discussion in section 6).

\subsection{Power coefficient validation}

In order to further validate the numerical tool, we compared the performance $\left(C_{P}\right)$ on the $B B M C$ configuration as a function of the TSR for different set angles to the numerical and experimental data from literature [1]. Concerning the experimental procedure, many details can be found in [1]. As regards the numerical results, they were obtained using two numerical tools based on the Blade Element Momentum (BEM) theory and reproduced from [1] too. The first numerical tool is a commercial code GH-Tidal Bladed (See for instance $[1,41]$ ) and the second one, SERG-Tidal is academic software developed by the Sustainable Energy Research Group (SERG) of Southampton university (See for instance [1, 14]). A single mesh discretisation (corresponding to $\varepsilon=0.075$ ) is presented since we have shown that the results are converged for this discretisation (cf. section 4.1).

For the four different set angles, Figure 11 shows that the $C_{P}$ results are in accordance with literature for the lower $T S R$ (i.e. $T S R \lesssim 4.0$ ), in the ascending phase 


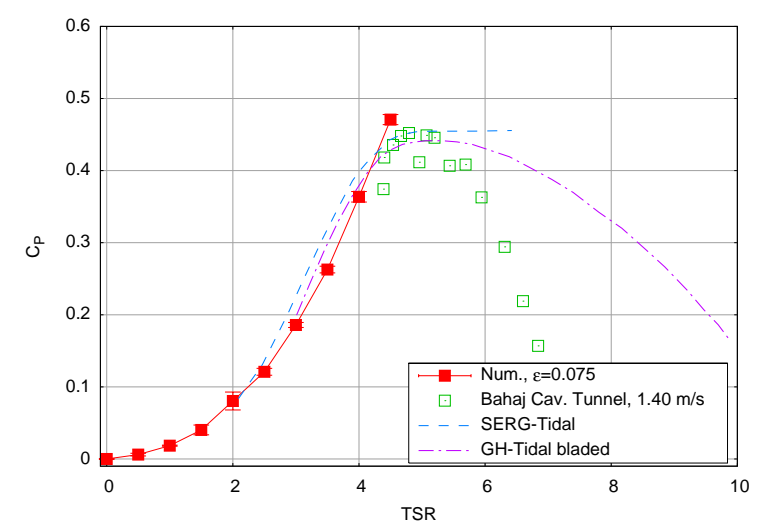

(a) $0^{\circ}$ set angle

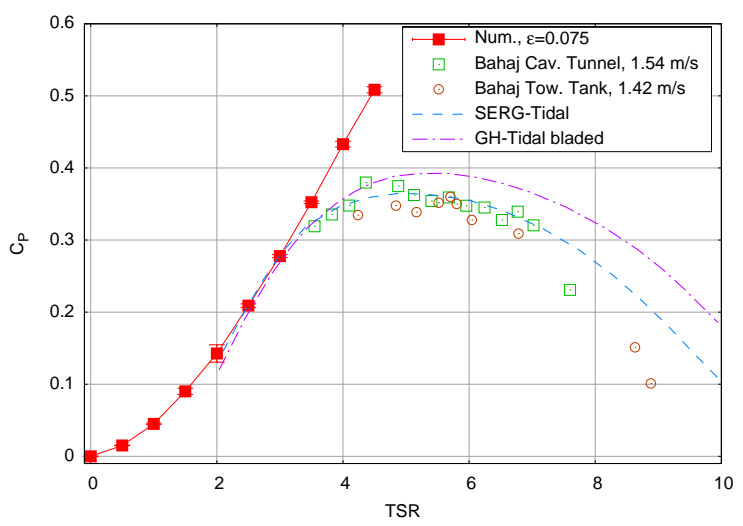

(c) $10^{\circ}$ set angle

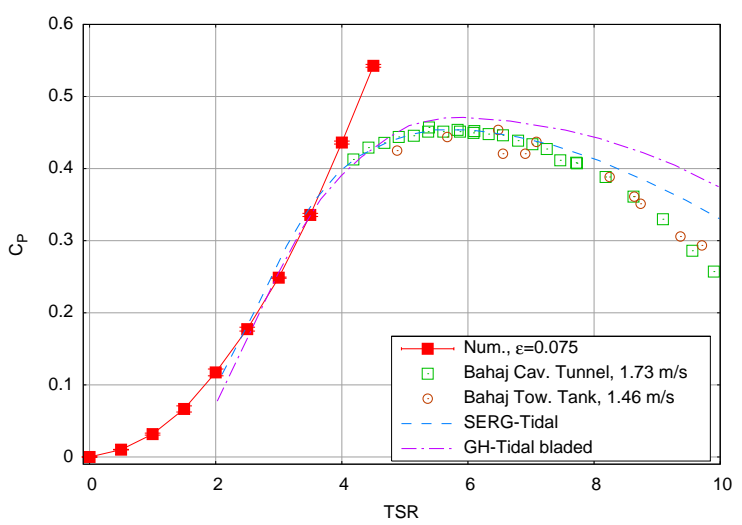

(b) $5^{\circ}$ set angle

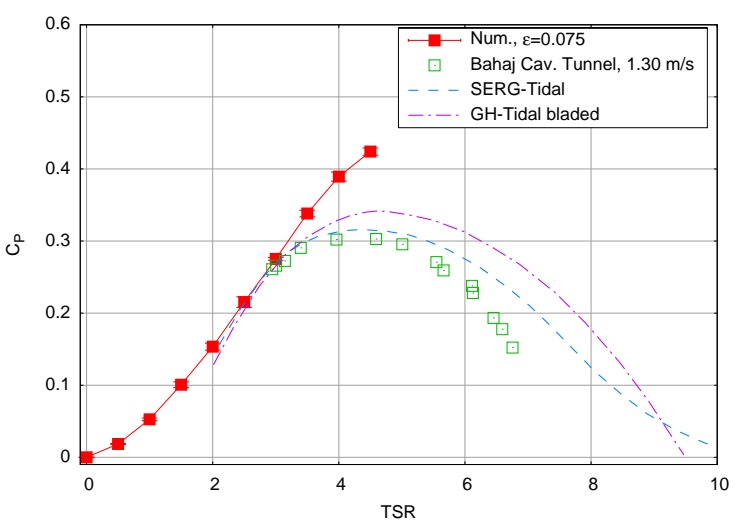

(d) $13^{\circ}$ set angle

Figure 11: Comparison of performance $\left(C_{P}\right)$ on the $B B M C$ configuration function of the TSR for different set angles (Cf. Table 1). Mean values are presented with the symbols and standard deviation as error bars. The GH-Tidal bladed, SERG-Tidal results and experimental datas have been reproduced from Bahaj et al. [1].

of the $C_{P}$ curve. For larger $T S R$, the $C_{P}$ values carry on increasing erroneously. This behaviour was expected as the present method is not able to correctly treat the flow separation that occurs where the local angle of attack becomes too high. From $4.0 \lesssim T S R \lesssim 6.0$, flow separation occurs on a larger part of the blade's span, and beyond $T S R \gtrsim 6.0$ flow separation occurs almost along the whole span. The problem was discussed in section 2.3 and work currently being carried out on a modified version of the particle emission in order to take into account the flow separation.

For the IFREMER-LOMC configuration, both performance $\left(C_{P}\right)$ and thrust $\left(C_{T}\right)$ were evaluated as a function of the TSR. A single mesh discretisation $(\varepsilon=$ $0.075)$ is presented. Comparisons of the numerical and experimental results are presented in Figure 12. The experimental data was obtained in the IFREMER flume tank of Boulogne-sur-Mer [42]. The experimental set-up is presented in detail in Maganga et al. [2] and the experimental trials were carried out with the same experimental procedure. Concerning the power coefficient $\left(C_{P}\right)$, from the results presented in Figure 12, the same observations as in the previous case (cf. Figure 11) can be made: the numerical $C_{P}$ values are in very good agreement with the experiments in 


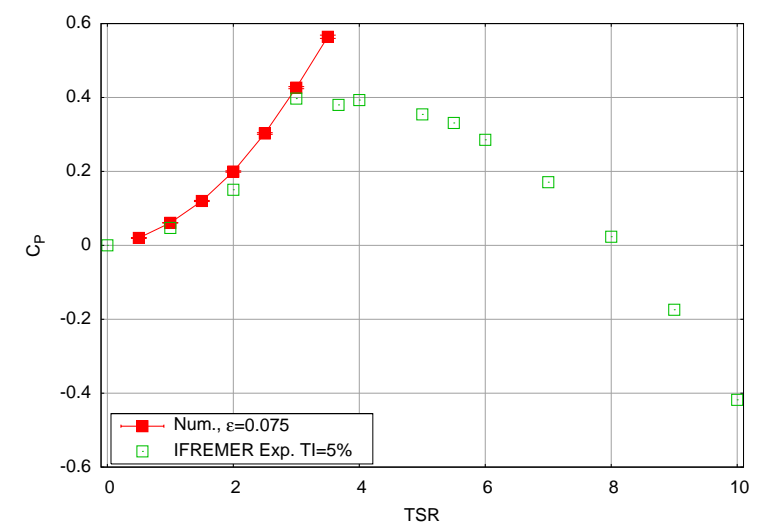

(a) $C_{P}$

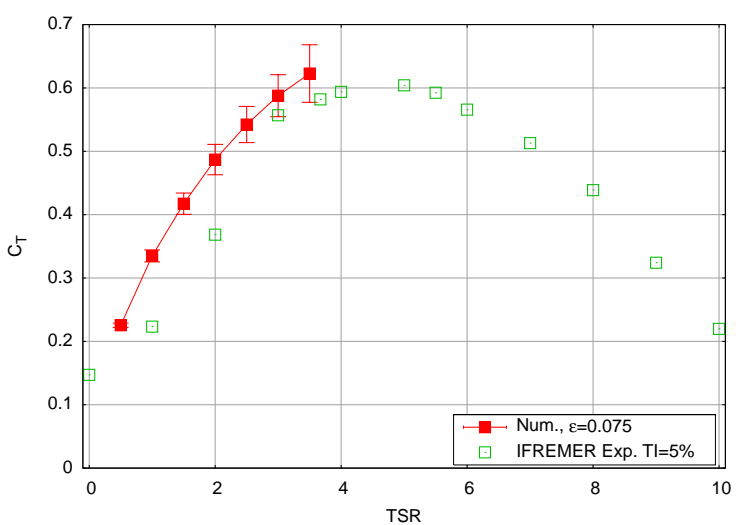

(b) $C_{T}$

Figure 12: Comparison of performance $\left(C_{P}\right)$ and thrust $\left(C_{T}\right)$ on the IFREMER$L O M C$ configuration function of the TSR. Mean values are presented with the symbols and standard deviation as an error bars. The experimental data wase obtained in the IFREMER flume tank of Boulogne-sur-Mer [42].

the validity range, that is in the ascending phase of the $C_{P}$ curve (i.e. TSR $\lesssim 3.5$ in that configuration). However, it is important to notice that the numerical tool is consistent and correctly reproduces the turbine performance whatever the geometry and the set angles are. As far as the thrust coefficient $\left(C_{T}\right)$ presented in Figure 12 is concerned, the numerical results are also in accordance with the experiments. However, the standard deviation of the results are much larger than they are for the power coefficient. This indicates that the drag force $\mathcal{F}_{x}$ oscillates around the mean value with a higher amplitude than for the $x$-moment. Consequently, the turbine might be more stressed in terms of fatigue due to this dynamic effect than it would be if only the static effect was considered. The range of validity (i.e. TSR $\lesssim 3.5$ ) is about the same as for the performance coefficient $\left(C_{P}\right)$, even slightly larger (with $T S R \approx 4.0, C_{T}$ is still correctly reproduced).

\section{Marine current turbine wake characterisation}

To have a complete characterisation of the wake, computations have to be run on a longer total physical time (i.e. $\Delta t_{t o t}=30.0 \mathrm{~s}$ ) than for the $C_{P}$ and $C_{T}$ evaluation, in order to let the wake develop correctly over a distance of more than 10 diameters behind the turbine. In addition, an average has to be computed on the concerned velocities over $\Delta t_{m}=4.0 \mathrm{~s}$ in order to have mean values. Figure 13 presents the CPU time consumptions for the different discretisations (see Table 3 and Table 4 for details) as well as the total number of particles in abscissae. The computations were run on a parallel architecture, using MPI libraries, in CRIHAN (Centre des Ressources Informatiques de HAute-Normandie), on 16 nodes of bi-processors quadcores Intel Nehalem EP @ 2,8 GHz.

Some general remarks and comments can be drawn from the data presented in Figure 13. Firstly, a finer discretisation leads to an increasing number of particles. Consequently, the finer the discretisation is, the more CPU time consuming 


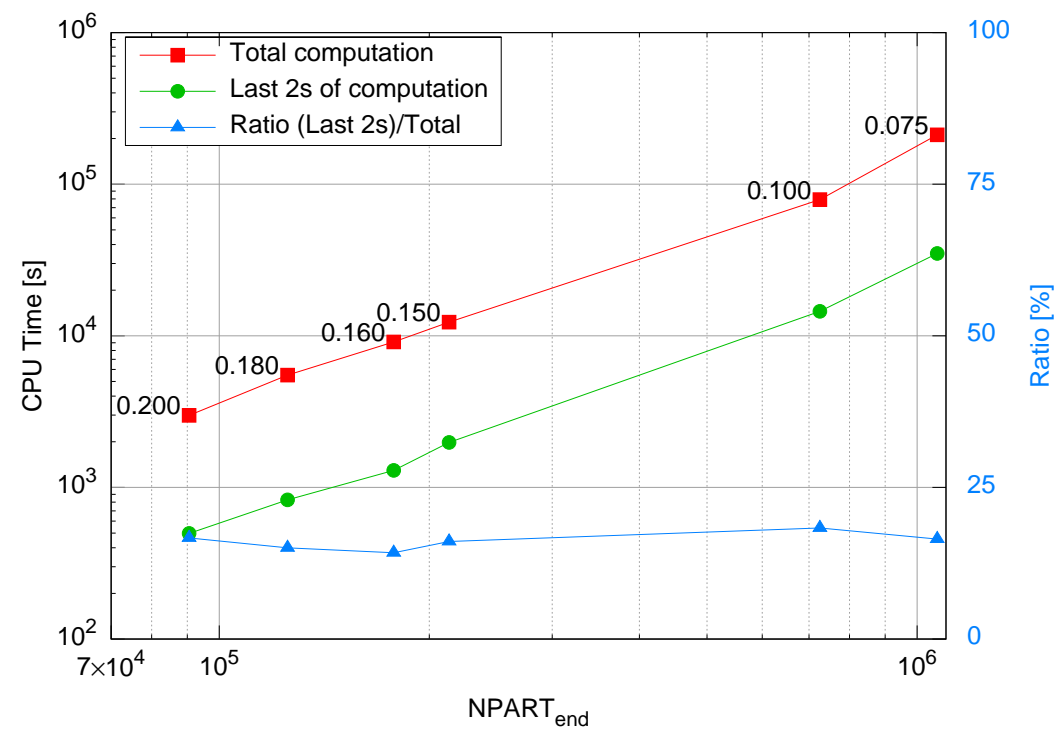

Figure 13: CPU time consumption as a function of the total number of particles $\left(N P A R T_{\text {end }}\right)$, corresponding to different discretisations $\varepsilon$ (see Table 3 and Table 4 ). The CPU times are given in seconds and obtained using 16 nodes of bi-processors quad-cores Intel Nehalem EP @ 2,8 GHz. $\varepsilon$ is indicated in the graph by the numerical values close to the red squares. Red squares indicate the total CPU time consumption for the computation of $\Delta t_{t o t}=30.0 \mathrm{~s}$ physical time. Green disks represent the CPU time consumption for the last $2 s$ physical time and blue triangles indicate the CPU percentage of these last $2 s$ physical time over a total elapsed time of $\Delta t_{\text {tot }}=30.0 \mathrm{~s}$.

the computations are. In fact, the coarsest discretisation $(\varepsilon=0.200)$ only uses approximately 90,000 particles with less than 3,000 $s$ of $\mathrm{CPU}(\approx 50$ minutes), whereas the finest discretisation $(\varepsilon=0.075)$ implies more than one million particles with approximately $212,000 s$ of $\mathrm{CPU}(\approx 2$ days and 11 hours). The problem being that with the two last discretisations $(\varepsilon=0.100$ and $\varepsilon=0.075)$, more and more small scales appear in the unsteady flow, which enhances its unsteadiness. As a conclusion, averaging over $\Delta t_{m}=4.0 \mathrm{~s}$ becomes insufficient to obtained a statistically correct mean value of the velocity components. A longer $\Delta t_{m}$ could have been used for the average, together with a longer $\Delta t_{\text {tot }}$. However, these computations being already the two most CPU consuming, computations on a longer $\Delta t_{\text {tot }}$ are not accessible yet, keeping in mind that the last physical seconds of computation are the most CPU consuming. In fact, whatever the discretisation is, computation of the last $\Delta t=2 \mathrm{~s}$ physical time approximately consumes $20 \%$ of the total CPU time. For these reasons, the wake computation analysis was performed on a new set of discretisations as described in Table 4, inspired from the previous Table 3. The new discretisations were obtained to fractionate the interval between $\varepsilon=0.200$ and $\varepsilon=0.150$.

\subsection{Characterisation of the wake}

Following the above discussion, this paragraph presents the numerical wakes for the new discretisations presented in Table 4 and compares them with experimental results [42]. The experimental trials were carried out in the same configuration as 


\begin{tabular}{cccccccc}
$\varepsilon$ & $d h$ & $N_{T E}$ & $l_{T E}$ & $N_{h u b}$ & $l_{h u b}$ & $d t(T S R=3.67)$ & CPU time (s) \\
\hline 0.200 & 0.133 & 5 & 0.156 & 6 & 0.128 & 0.035 & 2,986 \\
0.180 & 0.120 & 6 & 0.130 & 7 & 0.110 & 0.031 & 5,508 \\
0.160 & 0.106 & 7 & 0.111 & 8 & 0.096 & 0.028 & 9,108 \\
0.150 & 0.100 & 7 & 0.111 & 8 & 0.096 & 0.026 & 12,312
\end{tabular}

Table 4: Mesh description for different values of $\varepsilon$ for the wake computations. Geometrical description of these values can be found in Figure 4. The CPU times are given in seconds for a total physical time $\Delta t_{t o t}=30.0 \mathrm{~s}$ and obtained using 16 nodes of bi-processors quad-cores Intel Nehalem EP@2,8 GHz.

detailed in [2], with the same experimental procedure. Complete details of the turbine geometry, the IFREMER-LOMC configuration, are presented in section 3.1: one can refer to Table 1 for a general description and Table 2 for the blade description. Here, TSR $=3.67$ is considered since it is in the range of validity of our emission model in the $C_{P} / C_{T}$ curve (see Figure 12). As far as the ambient Turbulent Intensity ratio $(T I)$ is concerned, it has to be noted that the minimum reachable value for the experimental $T I$ was $5 \%$ whereas the numerical $T I$ was assumed to be zero, comparable to experimental conditions in a towing tank. As mentioned above, the computational tool is not yet able to take into account an ambient turbulent intensity ratio properly. Although the experimental TI is rather weak, with only $5 \%$, this might be a first explanation of the discrepancies in the results. New trials are already scheduled with different $T S R$ and $T I$, the ambient Turbulent Intensity ratio, and will be published in the near future.

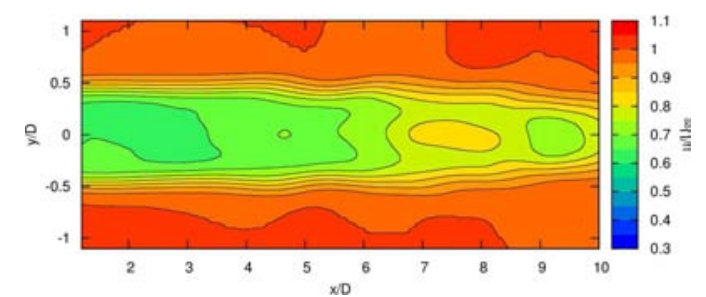

(a) Axial velocity, $\varepsilon=0.200$

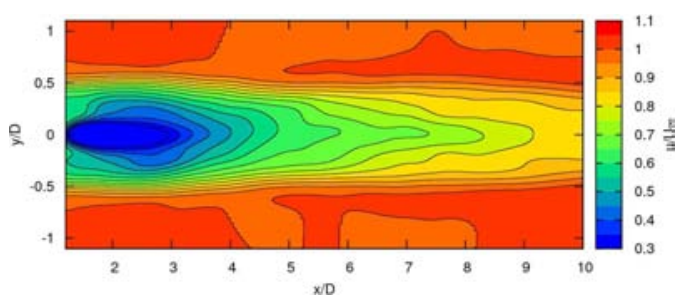

(c) Axial velocity, $\varepsilon=0.150$

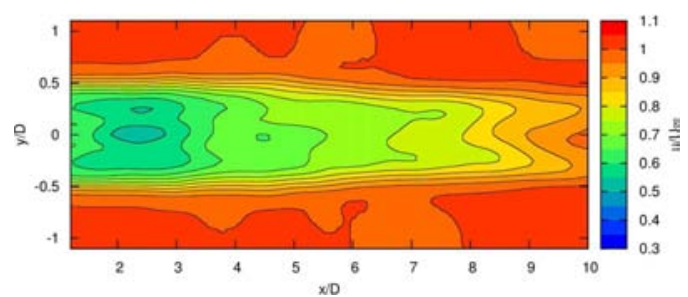

(b) Axial velocity, $\varepsilon=0.160$

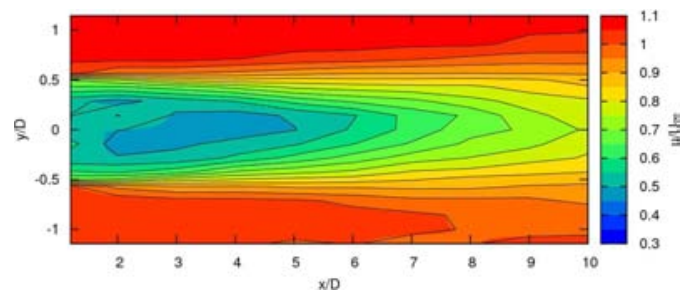

(d) Axial velocity, IFREMER experimental data, $\mathrm{TI}=5 \%$

Figure 14: Numerical mean axial velocity maps for $\varepsilon=0.2(14 \mathrm{a}), \varepsilon=0.16(14 \mathrm{~b})$, $\varepsilon=0.15$ (14c) and the experimental axial velocity map with an ambient turbulence intensity (TI) of $5 \%(14 \mathrm{~d})$.

Figure 14 shows the different mean axial velocity $u / U_{\infty}$ maps corresponding to 
different numerical discretisations, the last graph (Fig. 14d) corresponding to the experimental one. At first sight, the general shape of the wake is well reproduced if compared to the experimental one, whatever the discretisation is and despite the difference in ambient turbulence intensity rates. However, a better observation of the results leads to three main remarks.

The first remark resides in the difference between the experimental and the numerical results, of the lateral expansion of the wake basically defined here as the locus where $u / U_{\infty}=1.0$. In fact, the experimental lateral expansion of the wake is approximately $1.5 D$ at $x / D=10.0$ (i.e. between $y / D=-0.75$ and $y / D=+0.75$ ). At the same location in the numerical wake $(x / D=10.0)$, for $\varepsilon=0.200, \varepsilon=0.160$ and $\varepsilon=0.150$, this lateral expansion is approximately $1.0 \mathrm{D}$. These observations clearly indicate that the turbulence model is at fault. In fact, as presented in section 2.2 equation (27), the eddy viscosity $\nu_{\text {turb }}$ is homogeneous whereas wake flows are characterised by their directionality aligned with the free stream. This problem of turbulence model will also be discussed in the conclusion.

Secondly, the numerical wake shapes are somehow wavy, which is very easily observable in Figure $14 \mathrm{c}$ between $x / D=5.0$ and $x / D=9.0$. This general wavy aspect is not present in the experimental results (Figure 14d). This difference can be attributed to the unsteady property of the numerical results: even after an average over $\Delta t_{m}=4.0 \mathrm{~s}$, the mean velocity does not seem to be completely statistically correct. In fact, the computed flow becomes more and more turbulent (and thus more and more unsteady) as the discretisation size decreases. Thus, the finer the discretisation is, the more important the influence of the turbulence model is (cf. section 2.2). The unsteadiness of the flow being more important, averaging operations could have been applied on longer times than $\Delta t_{m}=4.0 \mathrm{~s}$. That is to say, the complete computation has to be computed on longer time than $\Delta t_{\text {tot }}=30.0 \mathrm{~s}$, which is not accessible at the present time with the present numerical implementation. The lack of averaging is actually the main reason for this wavy behaviour of the computed wake.

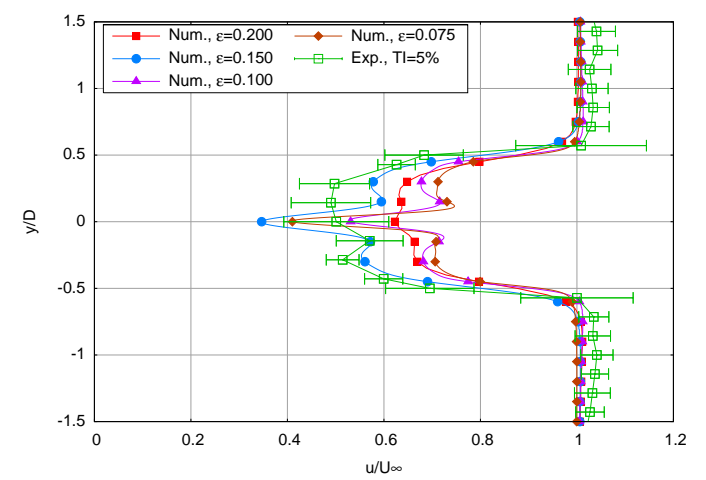

(a) $x=1.2 D$

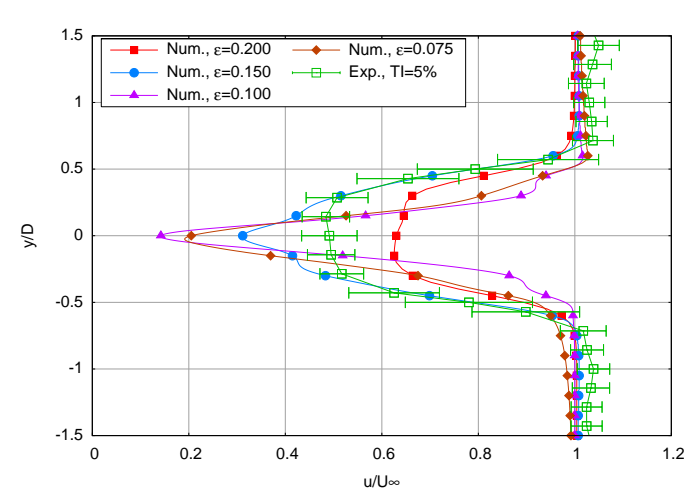

(b) $x=3.0 D$

Figure 15: Axial velocity profiles at two different locations in the near wake of the turbine for different $\varepsilon$ values (see Table 3).

From the graphs presented in Figure 14, the final remark would be the difference 
in the maximum velocity deficit just behind the turbine, for $x / D \lesssim 4.0$. In the experiments, the near wake flow is a combination of three wakes: the hub wake, the mast wake and the rotating blade wake. The asymmetry in the experimental near wake (Fig. 14d) can clearly be attributed to the interaction of the rotating blade wake with the fixed-mast wake. In the computations, only the hub and the blades are taken into account, which may be one explanation. Furthermore, the minimum axial velocity (inversely proportional to the maximum axial velocity deficit) is approximately $u / U_{\infty} \approx 0.3$ for the numerical computations (Fig. 14c) whereas it is only $u / U_{\infty} \approx 0.5$ in the experiments (Fig. 14d). This discrepancy is rather difficult to analyse because the flow is very complex in this region. Figure 15 depicts the numerical velocity profiles for two locations in the near wake $(x / D=1.2$ and $x / D=3.0$ ) for the first set of discretisations (see Table 3). On the numerical profiles presented in Figure 15a, the hub wake and the blades wake are easily distinguishable, whereas the experimental wake is already well mixed. At one diameter behind the blades, the turbulent mixing is already not well reproduced numerically. At three diameters downstream (Fig. 15b), the numerical maximum velocity deficit converges around an approximate value of $u / U_{\infty} \approx 0.2$ against $u / U_{\infty} \approx 0.5$ in the experiments. The conservation of mass is not at fault but the lack of mixing is even strengthened with finer discretisations $(\varepsilon=0.100$ and $\varepsilon=0.075)$ : the computations do not correctly recover the Gaussian velocity deficit. As already mentioned, the weakness of our turbulence model is highlighted. For the two lowest discretisations ( $\varepsilon=0.100$ and $\varepsilon=0.075$ ), the far wakes are not presented owing to the previous discussion at the beginning of section 5. In following section 5.3, the axial velocity deficit will be discussed in more depth.

\subsection{Wake velocity profiles}

From the maps presented on Figure 14, a closer look can be taken by considering the velocity profiles at different particular locations behind the turbine, even far behind the wake at eight diameters downstream. Figure 16 depicts these velocity profiles taken at $x / D=1.2,2.0,3.0,4.0,5.0,6.0,7.0$ and 8.0. The first profile could only be taken at $x / D=1.2$ for experimental reasons.

Several comments arise from the numerical velocity profiles presented in Figure 16; which may emphasise the previous remarks. Firstly, on Figure 16a, the numerical velocity profiles show a minimum velocity value well-marked at the profile centre (i.e. $y / D=0$ ). This well-marked shape can clearly be attributed to the hub wake, which at this point, has not yet mixed with the blade wake; the corresponding experimental velocity profile is more or less flat with $u / U_{\infty} \approx 0.5$ between $y / D=-0.45$ and $y / D=+0.45$. On Figures $16 \mathrm{c}$ and $16 \mathrm{~d}$, the mixing has now occurred in the numerical velocity profiles, at least for $\varepsilon=0.160$ and $\varepsilon=0.150$. The finest discretisation $(\varepsilon=0.150)$ is however the only one to correctly reproduce the Gaussian velocity profile for $x / D \geq 4.0$ (Fig. 16d to 16h). This lack of mixing is clearly a sign of deficiency of our turbulence model, as mentioned above. Smaller discretisations $(\varepsilon<0.150)$ may improve the results and will be tested in the future together with a better turbulence model.

For the far wake $(x / D \geq 4.0$, Fig. $16 \mathrm{~d}$ to $16 \mathrm{~h})$, the computed velocity profiles 


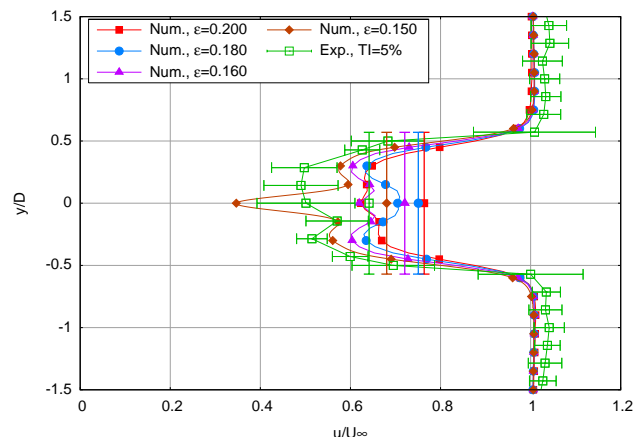

(a) $x=1.2 D$

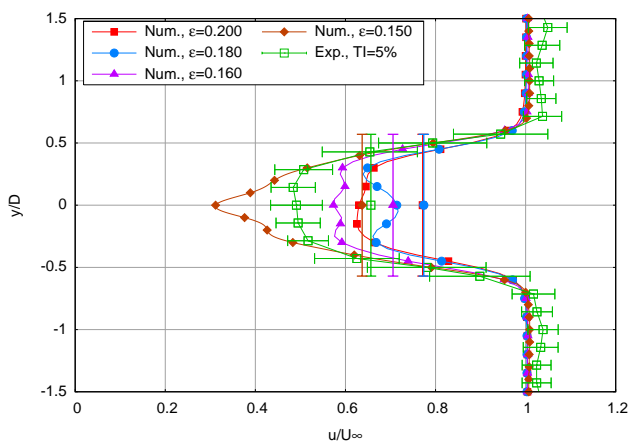

(c) $x=3.0 D$

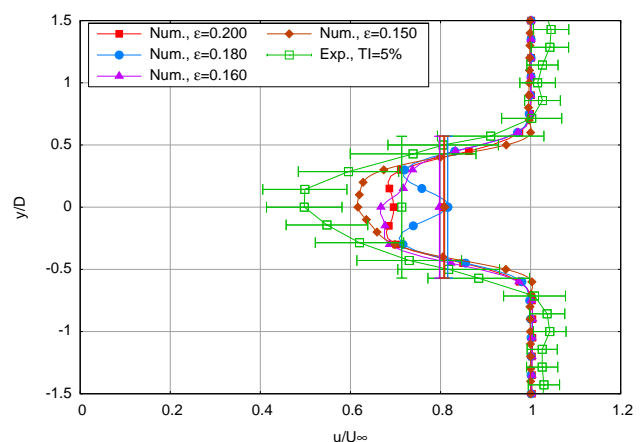

(e) $x=5.0 D$

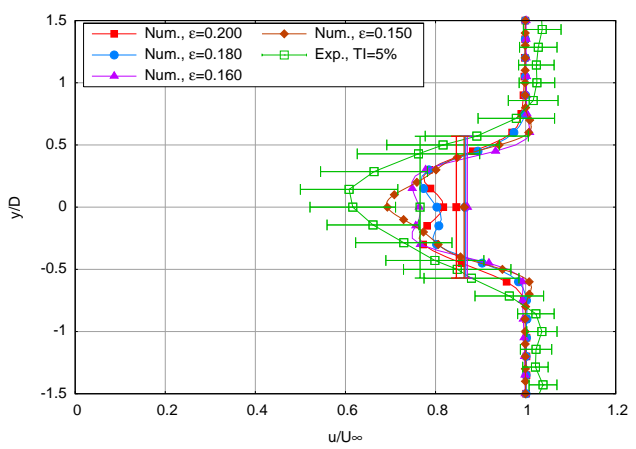

(g) $x=7.0 D$

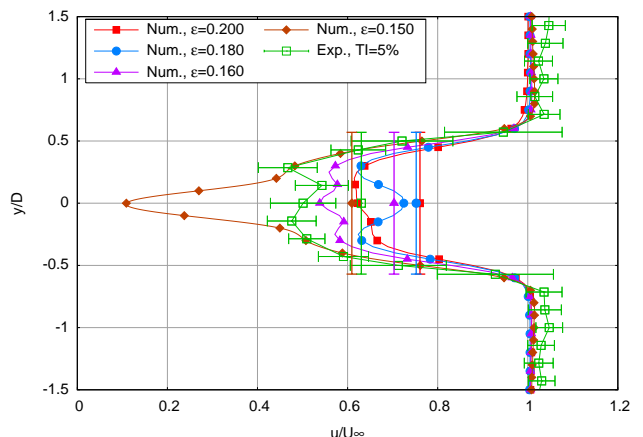

(b) $x=2.0 D$

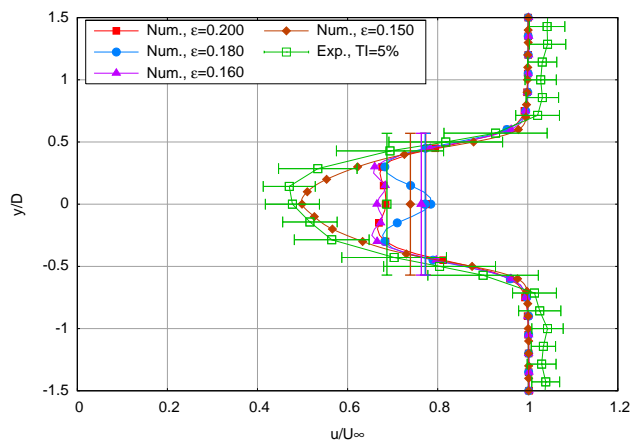

(d) $x=4.0 D$

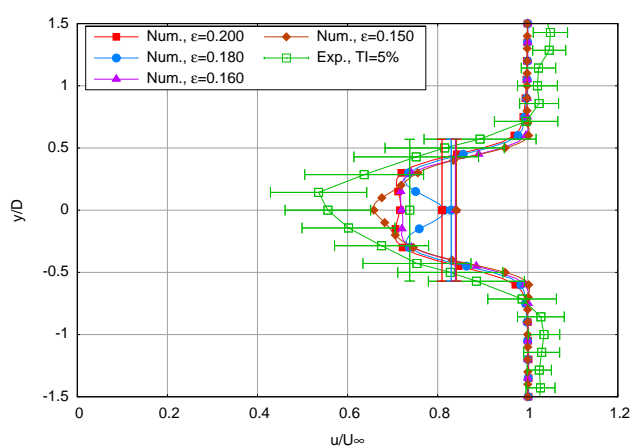

(f) $x=6.0 \mathrm{D}$

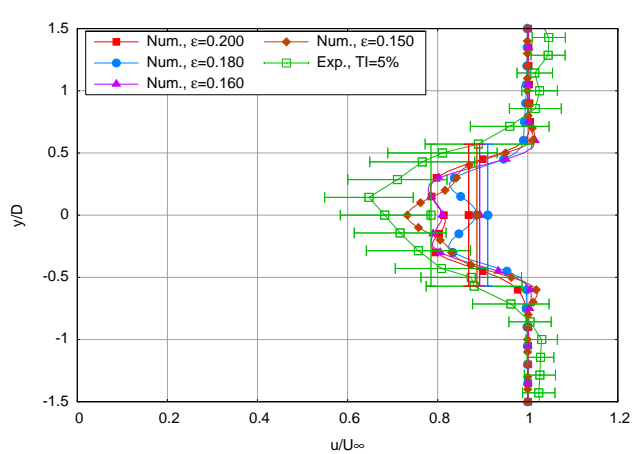

(h) $x=8.0 D$

Figure 16: Axial velocity profiles at different locations behind the turbine for different $\varepsilon$ values as described in Table 4 . The lateral bars for the experimental data represent the standard deviation around the mean velocity value. The vertical bars indicate the mean axial velocity value $\bar{u}(x)$ as computed with equation (51), section 5.3 . 
are very close to the experimental ones for all the discretisations. One disadvantage should be mentioned, despite the fact that the computed profiles are close, they all show a lower deficit than expected.

The conservation of mass is not at fault here, and the mean velocity deficit (indicated by the vertical bars on Figure 16 and computed as in section 5.3) could be a good indicator. In fact, in the near wake $(x / D \leq 3.0$, Fig. 16a to $16 \mathrm{c})$, the mean velocity deficits are closer to the experimental one as the discretisation decreases. At $x / D \approx 4.0$ (Fig. $16 \mathrm{~d}$ ), the mean velocity deficits are barely identical whatever the discretisations are and remain very similar in the far wake $(x / D \geq 4.0$, Fig. $16 \mathrm{~d}$ to $16 \mathrm{~h})$. Section 5.3 will treat this aspect in detail.

\subsection{Integrated axial velocity deficit}

From these profiles, the mean value of the axial velocity $\bar{u}(x)$ was estimated at position $x$ integrated on a $R^{*}=R+\delta r$ radius disc:

$$
\bar{u}(x)=\frac{1}{R^{* 2}} \int_{-R^{*}}^{R^{*}}|y| u(x, y) d y .
$$

Here we take $\delta r=0.05 \mathrm{~m} \simeq 0.14 R$, which enlarges the integration interval to the two nearest experimental measurement nodes outside the rotor. In this way, the whole velocity deficit is taken into account. The $R^{*}$ radius disc thus represents the turbine's area of influence, which is slightly larger than the turbine's cross-section area. This mean axial velocity $\bar{u}(x)$ is very interesting in the perspective of several rows of marine current turbines in a farm. In fact, it approximately indicates the total amount of power $\left(\approx \frac{1}{2} \rho \pi R^{2} \bar{u}(x)^{3}\right)$ still present in the wake, keeping in mind that the velocity profiles are not homogeneous and very turbulent. Experimental studies are focusing on these configurations of several rows of marine current turbines, amongst others [42].

Now that the evaluation of the axial velocity mean value on the turbine's area of influence has been defined, we can examine the reduction of the velocity deficit as the distance from the turbine increases. The mean axial velocity deficit $\gamma$ (in \%) at a specific location $x$ behind the turbine is defined as

$$
\gamma(x)=100(1-\bar{u}(x))
$$




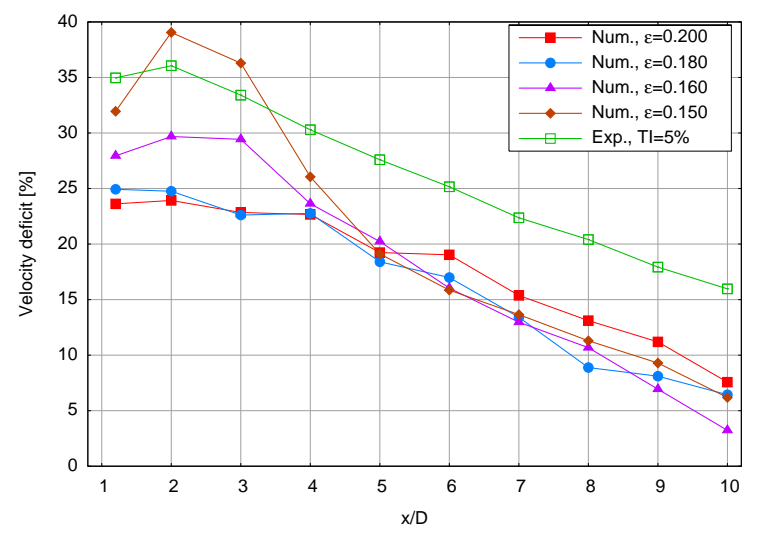

Figure 17: Mean axial velocity deficit $\gamma$ (in \%) as computed with equation 52 for the numerical computations for different $\varepsilon$ values (Table 4) compared to the experimental value with an ambient turbulence intensity (TI) of $5 \%$.

Figure 17 depicts the mean axial velocity deficit $\gamma$ (in \%) as computed with equation (52). Results of the four computations for different $\varepsilon$ values (Table 4) are compared to the experimental value with an ambient turbulence intensity $T I=5 \%$. As observed earlier, two different behaviours in the wake can be observed. The near wake is characterised by a velocity deficit, which is approximately constant and the far wake, where the velocity deficit decreases more or less linearly until zero, indicating that the initial velocity value has been recovered. First, the main difference between the numerical results and the experiments resides in the fact that the transition between the near and far wake does not occur at the same location. In fact, it occurs very close to the turbine in the experiments, approximately at $x / D \approx 2.0$, and only around $x / D \approx 3.0 \sim 4.0$ for the numerical results depending on the resolution $(\varepsilon)$. Once again, this behaviour is mainly due to a lack of mixing as discussed in section 5.2 and above.

Secondly, for the near wake, the finer the resolution is, the higher the velocity deficit. And the closer to the experimental results it gets. The increase in velocity deficit between $x / D=1.2$ and 2.0, very strong for $\varepsilon=0.150$ but also present in the experiments, is only due to radial effects. In fact, the mean velocity deficit $\gamma$ is only computed for a disc of radius $R^{*}$. For the numerical far wake $(x / D \gtrsim 3.0 \sim 4.0)$, all the computations almost superimpose themselves but not with the experimental curve. Two main comments can be made: there is a gap ab initio, the velocity deficit $\gamma$ is always inferior to the experimental one (except for $\varepsilon=0.150$ but it decreases very rapidly between $x / D=3.0$ and 4.0 ) and the slope of decrease is higher in the numerics than in the experiments. A higher slope can clearly be attributed to a higher dissipation than expected in the numerical scheme. Here again, the turbulence model can be blamed but not alone. A higher order integration scheme than the present $2^{\text {nd }}$ order Runge Kutta or a higher order smoothing function for the kernel regularisation (eq. (8)) may enhance the quality of the results.

\section{Conclusion}

Numerical computations of three bladed horizontal axis marine current turbines were presented on both power and thrust coefficient $\left(C_{P}\right.$ and $\left.C_{T}\right)$ predictions as well 
as wake characterisation, up to ten diameters downstream of the turbine. Concerning the $C_{P}$ and $C_{T}$ predictions, a disadvantage has to be mentioned in that only the ascending phase of the $C_{P} / C_{T}$ curves can be modelled at the present time. In fact, for large $T S R$-values, flow separation cannot be treated with the present numerical implementation and this is going to be one of the two major developments in the near future. However, in the TSR validity range of the method, a convergence analysis study was performed for two different marine current turbines configurations. It shows that the numerical tool gives accurate results if compared to literature [1, 42]. Moreover, the present implementation is consistent as it gives accurate results for the two different configurations, a thin and elongated blade shape for the $B B M C$ configuration [1] and a larger chord slightly thicker shape, for the IFREMER-LOMC one [42]. Therefore, the expected results should be as accurate for other blades' configuration, in the limit of moderate relative thickness $(t / c)$ and $T S R$ validity range.

Regarding the wake characterisation, computations of unsteady 3D wake flows were run in this study. Velocity maps and profiles in the wake of the turbine are in good qualitative and quantitative accordance with experiments, up to ten diameters downstream of the turbine. However, owing to a growing CPU time consumption as the computation resolution is refined, the wake flow could not be obtained for the finest resolutions (i.e. $\varepsilon \lesssim 0.100$ ). A better parallel implementation together with the use of more than the current 128 cores may make these computations accessible within a reasonable duration. Nevertheless, the implementation of a better turbulence model is compulsory as the present one fails in many points: insufficient mixing behind the turbine hub, insufficient diffusion as the lateral extension of the wake indicates and finally too high a numerical dissipation as highlighted by the axial velocity deficit. Several other turbulence models are available in literature (see for instance $[35,36]$ ) and this will be the other major development of the numerical tool for the future. In addition, the account of different ambient turbulence intensity (TI) rates would be of great interest if one wants to get a better evaluation of the wake flow (and thus the global flow perturbation) of a marine current turbine in real conditions, with more realistic ambient turbulence intensity (TI) rates. Moreover, several TI rates are available in the IFREMER flume tank of Boulogne-sur-Mer [2], which may form an interesting starting point for numerical/experimental comparisons.

As short-term perspectives, numerical tests could be run with the present tool for other blade configurations, with or without a duct. The wake characterisation for different $T S R$ could be evaluated in the perspective of marine current turbines in a farm. With respect a turbine farm, another short term perspective would be to run computations of wake interactions between two or three turbines. No real important modification in the software is required, as the present numerical scheme can easily handle several turbines provided that the CPU performance is and/or an access to larger CPU resources is granted. Experimental results on turbine interactions are also already available [42] to validate these future computations. For long-term perspectives, when a new particles emission scheme and a better turbulence model are implemented, more complex configuration could be foreseen: a boundary layer profile in the upstream velocity, wave and current upstream velocity and finally, more realistic sea states combining the two previous cases together with realistic 
ambient turbulence intensity rates.

\section{Acknowledgements}

The authors would like to thank Haute-Normandie Regional Council for their financial supports for co-financed PhD theses and the RHYNO project. The authors also would like to thank the CRIHAN (Centre des Ressources Informatiques de HAute-Normandie) for their available numerical computation resources. We are also grateful to Thomas Bacchetti, Jean- Matthieu Etancelin, Jean-Valery Facq, Benoit Gaurier and Fabrice Maganga for their help in the present work. The numerical software was initially developed for aeronautic applications as part of works with Aicrelle (SAFRAN group) and Haute-Normandie Regional Council.

\section{References}

[1] A. Bahaj, W. Batten, G. McCann, Experimental verifications of numerical predictions for the hydrodynamic performance of horizontal axis marine current turbines, Renewable Energy 32 (15) (2007) 2479 - 2490. doi:DOI: 10.1016/j.renene.2007.10.001.

URL http://www.sciencedirect.com/science/article/B6V4S-4R05BKN-1/2/6ce5b7d63

[2] F. Maganga, G. Germain, J. King, G. Pinon, E. Rivoalen, Flow characteristic effects on marine current turbine behaviour and on its wake properties, IET Renew. Power Gener. Vol. 4 (6) (2010) doi:10.1049/iet-rpg.2009.0205 - p. 498509 .

[3] M. Grabbe, E. Lalander, S. Lundin, M. Leijon, A review of the tidal current energy resource in norway, Renewable and Sustainable Energy Reviews 13 (2009) 1898-1909. doi:DOI: 10.1016/j.rser.2009.01.026.

[4] F. O. Rourke, F. Boyle, A. Reynolds, Marine current energy devices: Current status and possible future applications in ireland, Renewable and Sustainable Energy Reviews 14 (2009) 1026-1036. doi:DOI: 10.1016/j.rser.2009.11.012.

[5] D. Li, S. Wanga, P. Yuan, An overview of development of tidal current in china: Energy resource, conversion technology and opportunities, Renewable and Sustainable Energy Reviews 14 (2010) 2896-2905. doi:DOI: doi:10.1016/j.rser.2010.06.001.

[6] S. Wang, P. Yuan, D. Li, Y. Jiao, An overview of ocean renewable energy in china, Renewable and Sustainable Energy Reviews 15 (2011) 91-111. doi:DOI: 10.1016/j.rser.2010.09.040.

[7] L.S.Blunden, A. Bahaj, Initial evaluation of tidal stream energy resources at portland bill, uk, Renewable Energy 31 (2006) 121-132. doi:DOI: 10.1016/j.renene.2005.08.016.

[8] M. Shields, L. Dillon, D. Woolf, A. Ford, Strategic priorites for assessing ecological impacts of marine renewable energy devices in pentland firth (scotland, uk), Marine Policydoi:DOI: 10.1016/j.marpol.2008.12.013. 
[9] G. Trowse, R. Krasten, Bay of fundy tidal energy development - opportunities and challenges, Proceeding of the 3rd International Conference on Ocean Energy, Bilbao, Spain, October 6-8.

[10] J. Giles, I. Godfrey, I. Bryden, L. Myers, J. O’Nians, A. Bahaj, J. Griffiths, An innovative tidal fence development for severn estuary, Proceeding of the 3rd International Conference on Ocean Energy, Bilbao, Spain, October 6-8.

[11] S. P. Neill, E. J. Litt, S. J. Couch, A. G. Davies, The impact of tidal stream turbines on large-scale sediment dynamics, Renewable Energy 34 (2009) 28032812. doi:DOI: 10.1016/j.renene.2009.06.015.

[12] W. Batten, A. Bahaj, A. Molland, J. Chaplin, Experimentally validated numerical method for the hydrodynamic design of horizontal axis tidal turbines, Ocean Engineering 34 (2007) 1013 - 1020. doi:DOI: 10.1016/j.oceaneng.2006.04.008.

[13] X. Sun, J. Chick, I. Bryden, Laboratory-scale simulation of energy extraction from tidal currents, Renewable Energy 33 (2008) 1267-1274. doi:DOI: 10.1016/j.renene.2007.06.018.

[14] W. Batten, A. Bahaj, A. Molland, J. Chaplin, The prediction of hydrodynamic performance of marine current turbine, Renewable Energy 33 (5) (2008) 1085 - 1096. doi:DOI: 10.1016/j.renene.2007.05.043.

[15] J. Baltazar, J. F. ao de Campos, Hydrodynamic analysis of a horinzontal axis marine current turbine with a boundary element method, Proceeding of the ASME 27th International Conference on Offshore Mechanics and Artics Engineering, Estoril, Portugal, June 15-20.

[16] A. Bahaj, A. Molland, J. Chaplin, W. Batten, Power and thrust measurements of marine current turbines under various hydrodynamic flow conditions in a cavitation tunnel and a towing tank, Renewable Energy 32 (3) (2007) 407 426. doi:DOI: $10.1016 /$ j.renene.2007.10.001.

[17] L. Myers, A. Bahaj, R. Rawlinson-Smith, M. Thomson, The effect of boundary proximity upon the wake structure of horinzontal marine current turbines, Proceeding of the ASME 27th International Conference on Offshore Mechanics and Artics Engineering, Estoril, Portugal, June 15-20.

[18] M. Harrison, W. Batten, L. Myers, A. Bahaj, Comparison between cfd simulations and experiments for predicting the far wake of horizontal axis tidal turbines, IET Renew. Power Gener. 4 (6) (2010) 613-627. doi:DOI: 10.1049/ietrpg.2009.0193.

[19] S. Draper, G. Houlsby, M. Oldfield, A. Borthwick, Modelling tidal energy extraction in depth-avergaed coastal domain, IET Renew. Power Gener. 4 (6) (2010) 545-554. doi:DOI: 10.1049/iet-rpg.2009.0196.

[20] L. Myers, A. Bahaj, Experiment alanalysis of the flow field around horizontal axis tidal turbines by use of scale mesh disk rotor simulators, Ocean Engineering 37 (2010) 218 - 227. doi:DOI: 10.1016/j.oceaneng.2009.11.004. 
[21] S. Antheaume, T. Maître, J. Achard, Hydraulic darrieus turbines efficiency for free fluid flow conditions versus power farms conditions, Renewable Energydoi:DOI: $10.1016 /$ j.renene.2007.12.022.

[22] C. Consul, R. Willden, Influence of flow confinement on the performance of a crossflow turbine, Proceeding of the 3rd International Conference on Ocean Energy, Bilbao, Spain, October 6-8.

[23] G. Pinon, H. Bratec, S. Huberson, G. Pignot, E. Rivoalen, Vortex method for the simulation of a $3 \mathrm{~d}$ round jet in a cross-stream, J. Turbulence 6 (18) (2005) $1-25$.

[24] G. Pinon, Les jets d'Inverseurs de poussée d'un avion en phase d'atterrissage, Broché - Éditions Universitaires Europeénnes - ISBN-10: 6131508658 - ISBN13: 978-6131508653, 2010.

[25] F. Maganga, G. Pinon, G. Germain, E. Rivoalen, Wake properties characterisation of marine current turbine., 3rd International Conference on Ocean Energy (ICOE), Bilbao, Spain.

[26] K. Lindsay, R. Krasny, A particle method and adaptive treecode for vortex sheet motion in three-dimensional flow, J. Comput. Phys. 172 (2001) 879-907.

[27] G.-H. Cottet, P. D. Koumoutsakos, Vortex Method : Theory and Practice, Cambridge University Press, UK, 2000.

[28] J. L. Hess, A. M. O. Smith, Calculation of potential flow about arbitrary bodies, Progress in Aerospace Sciences 8 (1967) 1-138. doi:DOI: 10.1016/03760421(67)90003-6.

URL http://www.sciencedirect.com/science/article/B6V3V-4811PYT-33/2/053e7071

[29] J. Choquin, S. Huberson, Particles simulation of viscous flow, Computers \& Fluids 17 (2) (1989) 397-410.

[30] P. Degond, S. Mas-Gallic, The weighted particle method for convection-diffusion equations. Part I: The case of an isotropic viscosity, Math. Comput. 53 (1989) $485-508$.

[31] J. D. Eldredge, A. Leonard, T. Colonius, A general deterministic treatment of derivatives in particle methods, Journal of Computational Physics 180 (2) (2002) 686-709. doi:DOI: 10.1006/jcph.2002.7112.

URL http://www . sciencedirect.com/science/article/B6WHY-46G47HY-F/2/a01a8d80f

[32] E. Rivoalen, S. Huberson, The particle strength exchange method applied to axisymmetric viscous flows, J. Comp. Physics 168 (2001) 519-526.

[33] N. Mansour, J. Ferziger, W. Reynolds, Large-eddy simulation of a turbulent mixing layer, Report TF-11, Thermosciences Div., Dept. of Mech. Eng., Stanford University. 
[34] P. Sagaut, Large eddy simulation for incompressible flows: an introduction third edition.

[35] J. Mansfield, O. Knio, C. Meneveau, A dynamic LES scheme for the vorticity transport equation : formulation and a priori tests, J. Comp. Phys. 145 (Issue 2) (1998) 693-730.

[36] C. Meneveau, J. Katz, Scale-invariance and turbulence models for largeeddy simulation, Annual Review of Fluid Mechanics 32 (1) (2000) 1-32. arXiv:http://www.annualreviews.org/doi/pdf/10.1146/annurev.fluid.32.1.1, doi:10.1146/annurev.fluid.32.1.1.

URL http://www . annualreviews .org/doi/abs/10.1146/annurev.fluid.32.1.1

[37] F. Hauville, Optimisation des méthodes de calculs d'écoulements tourbillonnaires instationnaires, Ph.D. thesis, Université du Havre (1996).

[38] B. Marichal, F. Hauville, Numerical calculation af an incompressible, inviscid three-dimensional flow about a wind turbine with partial span pitch control, Société Roumaine de Mathématique appliquées et Industrielles - Oraéda, Roumanie.

[39] T. G. L. (http://www.tidalgeneration.co.uk/)[link].

URL http://www.tidalgeneration.co.uk/

[40] A. Bahaj, L. Myers, Analytical estimates of the energy yield potential from the alderney race (channel islands) using marine current energy converters, Renewable Energy 29 (12) (2004) 1931 - 1945. doi:DOI: 10.1016/j.renene.2004.02.013. URL http://www.sciencedirect.com/science/article/B6V4S-4C59RYG-2/2/0c5e6268b

[41] G.-T. B. (http://www.gl garradhassan.com/en/software/GHTidalBladed.php)[link]. URL http://www.gl-garradhassan.com/en/software/GHTidalBladed.php

[42] P. Mycek, B. Gaurier, G. Germain, G. Pinon, E. Rivoalen, Numerical and experimental study of the interaction between two marine current turbines, Proceedings of the 9th European Wave and Tidal Energy Conference (EWTEC), Southampton, UK. 